\title{
Obfuscation-Based Non-black-box Simulation and Four Message Concurrent Zero Knowledge for NP
}

\author{
Omkant Pandey ${ }^{1,2, \star, \star \star}$, Manoj Prabhakaran ${ }^{1, \star}$, and Amit Sahai ${ }^{2, \star \star}$ \\ 1 University of Illinois at Urbana Champaign, USA \\ \{omkant, mmp\}@uiuc. edu \\ 2 UCLA and Center for Encrypted Functionalities, USA \\ sahai@cs.ucla.edu
}

\begin{abstract}
We show the following result: Assuming the existence of public-coin differing-input obfuscation (pc-diO) for the class of all polynomial time Turing machines, then there exists a four message, fully concurrent zero-knowledge proof system for all languages in NP with negligible soundness error. This result is constructive: given pc-diO, our reduction yields an explicit protocol along with an explicit simulator that is "straight line" and runs in strict polynomial time. The obfuscation security property is used only to prove soundness.

Public-coin differing-inputs obfuscation is a notion of obfuscation closely related to indistinguishability obfuscation. Most importantly for our result, pc-diO does not suffer from any known impossibility results: recent negative results on standard differing-inputs obfuscation do not apply to pc-diO. Furthermore, candidate constructions for pc-diO for the class of all polynomial-time Turing Machines are known.

Our reduction relies on a new non-black-box simulation technique which does not use the PCP theorem. We view the development of this new non-black-box simulation technique as the main contribution of our work. In addition to assuming pc-diO, our reduction also assumes (standard and polynomial time) cryptographic assumptions such as collisionresistant hash functions.
\end{abstract}

\section{Introduction}

Zero-Knowledge and Program Obfuscation. Zero-knowledge proofs, introduced by Goldwasser, Micali and Rackoff [GMR85] are the classical example of the

\footnotetext{
* Research supported in part by the NSF Grant 1228856.

** Research supported in part from a DARPA/ONR PROCEED award, NSF Frontier Award 1413955, NSF grants 1228984, 1136174, 1118096, and 1065276, a Xerox Faculty Research Award, a Google Faculty Research Award, an equipment grant from Intel, and an Okawa Foundation Research Grant. This material is based upon work supported by the Defense Advanced Research Projects Agency through the U.S. Office of Naval Research under Contract N00014-11- 1-0389. The views expressed are those of the author and do not reflect the official policy or position of the Department of Defense, the National Science Foundation, or the U.S. Government.
}

Y. Dodis and J.B. Nielsen (Eds.): TCC 2015, Part II, LNCS 9015, pp. 638667 2015.

(C) International Association for Cryptologic Research 2015 
simulation paradigm. They allow a prover to convince a verifier that a mathematical statement $x \in \mathbf{L}$ is true while giving no additional knowledge to the verifier. Prior to 2001, all known zero-knowledge simulators used the (cheating) verifier $V^{*}$ as a black-box to produce their output (called the simulated view). Barak Bar01 demonstrated how to take advantage of verifier's program to build more powerful non-black-box simulation techniques.

Constructing and analyzing non-black-box simulators can be a challenging task.The reason why taking advantage of verifier's code is difficult is because of the intriguing possibility of program obfuscation. Roughly speaking, program obfuscation is a method to transform a computer program (say described as a Boolean circuit) into a form that is executable but otherwise completely "unintelligible." In its strongest form, an obfuscated program leaks no information about the program beyond its "functionality" or the "input-output behavior". Therefore, access to the obfuscated program is no better than having black box access to it. This property, as formalized by Barak, Goldreich, Impagliazzo, Rudich, Sahai, Vadhan, and Yang [BGI ${ }^{+}$01, is called the virtual black box (VBB) security. It was shown in $\mathrm{BGI}^{+} 01$ that VBB-secure obfuscation is impossible in general. In hindsight, this negative result shows why non-black-box (NBB) simulation is possible, despite the possibility that program obfuscation could hide nearly every useful aspect of the verifier's code.

Zero-knowledge, in particular non-black-box simulation, is intimately connected to program obfuscation. This connection has been explicitly studied in the works of Hada [Had00, and Bitansky and Paneth [BP12b, BP12a, BP13a, and alluded to in several other works, e.g., HT99, Bar01). In this work, we explore this line of research further, particularly in light of recent work showing the first plausible constructions of general-purpose obfuscation schemes $\left[\mathrm{GGH}^{+} 13\right]$. In particular, for the first time, we show that program obfuscation can be useful for designing new non-black-box simulation strategies that yield constant-round concurrent zero knowledge protocols.

General-Purpose Obfuscation. In 2013, Garg, Gentry, Halevi, Raykova, Sahai, and Waters $\mathrm{GGH}^{+} 13$ presented the first candidate construction for generalpurpose obfuscation. Several formalizations for obfuscation have been proposed as alternatives to the impossible-to-achieve notion of VBB obfuscation. A basic definition, called indistinguishability obfuscation (iO) [BGI ${ }^{+}$], roughly speaking, guarantees that if two (same-size) programs $C_{0}, C_{1}$ are functionally equivalent, then their obfuscations are computationally indistinguishable. A closely related notion is that of differing input obfuscation (diO) $\left[\mathrm{BGI}^{+} 01\right]$ which, roughly speaking, guarantees that the obfuscations of $C_{0}$ and $C_{1}$ are computationally indistinguishable provided that it is hard to find an input $x$ such that $C_{0}(x) \neq C_{1}(x)$. Unfortunately, recently evidence was shown BP13b, GGHW14 that the notion of $\mathrm{diO}$ is impossible to achieve in general, due to the existence of problematic contrived auxiliary inputs. However, very recently, Ishai, Pandey, and Sahai IPS15] formulated the notion of public-coin differing-inputs obfuscation (pc-diO) in which no auxiliary input is allowed except for the random coins of the sampler. This modification avoids the negative results of BP13b, 
GGHW14, and indeed all previous negative results on obfuscation using auxiliary input GK05, GK13, as all previous negative results using auxiliary input critically relied on the possibility of a secret being embedded within the auxiliary input. Because in pc-diO the auxiliary input is only allowed to be public randomness, this possibility is eliminated (please see [IPS15] for further details). Furthermore, [IPS15, building on [ABG+13, BCP14], present candidate constructions of pc-diO for the class of all polynomial-time Turing machines which can accept inputs of unbounded polynomial length.

Our Results. In this work we show how to use program obfuscation to build a new non-black-box simulation strategy that works for fully concurrent zeroknowledge. More specifically, we show that:

- If public-coin differing-input obfuscation (pc-diO) exists for the class of all polynomial time Turing machines with unbounded inputs, then there exists a constant round, fully concurrent zero knowledge protocol for NP with negligible soundness error. The protocol has an explicit simulator 1 the simulator is "straight line" and runs in strict polynomial time. The security of the obfuscation is used only prove the soundness of our protocol.

- We also show how to implement the core ideas of the above protocol in only four rounds. That is, our new protocol requires sending only four messages between the prover and the verifier.

Our protocol can be instantiated using the constructions of $\mathrm{BCP} 14, \mathrm{ABG}^{+} 13$, IPS15] which obfuscates polynomial time Turing machines that can accept inputs of variable length (at most polynomial in the security parameter). We stress that we are able to obtain an explicit simulator for our protocol irrespective of the computational assumptions underlying the constructions of differing-inputs obfuscation. This is because we use the security - i.e., the public-coin differinginputs security property - of obfuscation only in proving the soundness of our protocol. The simulator only depends on the correctness or the functionality of the obfuscated program, and hence can be described explicitly.

Other than pc-diO, our reduction only assumes standard (polynomial time hardness) assumptions, namely injective one-way functions and collision-resistant hash functions. Interestingly, our reduction does not explicitly depend on CSproofs or universal-arguments Kil92, Mic94, Kil95, BG02]; in particular, if we instantiate the constructions of IPS15, $\mathrm{ABG}^{+} 13$ using the SNARKs of Bitansky et al. [BCCT13] based on bilinear maps (which do not rely on the PCP theorem), we obtain an instantiation of our protocol that also does not rely on the PCP theorem.

The round complexity of our final protocol also sheds new light on the exact (as opposed to asymptotic) round-complexity of concurrent zero-knowledge.

${ }^{1}$ In some protocols, specifically those based on knowledge-type assumptions HT99, by virtue of the assumption that there exists an "extractor," it is only possible to obtain an existential result that a simulator exists; however, the actual program of the simulator is not explicitly given in the security proofs. 
Even in the simpler case of stand alone zero knowledge, the best known constructions require at least four rounds [FS89, and historically, concurrent zeroknowledge has always required more rounds than stand-alone zero-knowledge 2 Our four-round protocol, for the first time, closes the gap between the best known upper bounds on round complexities of concurrent versus standalone zero-knowledge protocols (whose simulators can be explicitly described).

In retrospect, the fact that obfuscation actually helps non-black-box simulation can be perplexing. Indeed, in all prior works along this line Had00, BP12b, BP13a, the core ideas for simulation are of opposite nature: it is the inability to obfuscate the "unobfuscatable functions" that helps the simulator. In our case, similar to BP12a, it is the ability to obfuscate programs that allows polynomial time simulation. We believe that our method can be useful in other settings as well where non-black-box simulation seems essential such as constant-round leakage-resilient zero-knowledge Pan14, GJS11 or CCA secure commitments in sub-logarithmic rounds $\mathrm{CLP} 10, \mathrm{GLP}^{+} 15$.

Paper Organization. We start by discussing how to use program obfuscation to avoid the use of universal arguments in Barak's protocol in Section 1.1. This results in a stand alone ZK protocol with a "straight line" simulator. In Section 4 , we discuss why the simulator of this protocol fails in the concurrent setting, and present a (substantially) different constant-round protocol which is concurrent ZK along with main proof ideas. In Section 5, we present an overview of our fourround concurrent-ZK protocol. The full details can be found in the full version of this work [PPS15].

\subsection{Technical Overview: Non-black-box Simulation via Program Obfuscation}

Let us start by considering the simplest approach to zero-knowledge from (the possibility of) program obfuscation. For now, let us restrict ourselves to the case of stand alone zero-knowledge for NP-languages. Let $x \in \mathbf{L}$ be the statement and $R$ be the witness-relation.

One simple approach is to have the verifier send an obfuscation of the following program $M_{x, s}$ which contains a secret string $s \in\{0,1\}^{n}: M_{x, s}(a)=s$ if and only $R(x, a)=1$ and $M_{x, s}(a)=0^{n}$ otherwise. Let $\widetilde{M}_{x, s}$ denote the iO-secure obfuscation of $M_{x, s}$. The real prover can recover $s$ by using a witness $w$ to $x$. Further, if $x$ is false, $M_{x, s}$ is identical to $M_{x, 0^{n}}$ and therefore must hide $s$, ensuring the soundness 3 This gives us a two-message, honest verifier ZK proof. However, this idea does not help the simulation against malicious verifiers.

To fix this, let us try to use Barak's preamble (called GenStat Bar01]) which has the following three rounds: first, the verifier sends a collision-resistant hash

\footnotetext{
${ }^{2}$ Barak's (bounded-concurrent ZK) protocol Bar01 and recent construction of Chung, Lin, and Pass CLP13b require at least six rounds even after optimizations; the recent protocol of Gupta and Sahai GS12b requires five rounds and does not have an explicit simulator.

${ }^{3}$ By security of iO, $\widetilde{M}_{x, s} \stackrel{c}{\approx} \widetilde{M}_{x, 0^{n}}$ and $\widetilde{M}_{x, 0^{n}}$ has no information about $s$.
} 
function $h:\{0,1\}^{*} \rightarrow\{0,1\}^{n}$, then the prover sends a commitment $c$ to $0^{n}$ (using a perfectly binding scheme Com), and then the verifier sends a string $r \in\{0,1\}^{n}$. The transcript defines a "fake statement" $\lambda=\langle h, c, r\rangle$. A "fake witness" $\omega$ for the statement $\lambda$ consists of a pair $(\Pi, u)$ such that $c=\operatorname{Com}(h(\Pi) ; u)$ and $\Pi$ is a program of length poly $(n)$ which outputs the string $r$ on input the string $c$ (say, in $n^{\log \log n}$ steps). If $h$ is a good collision-resistant hash function, then it was shown in Bar01, BG02, no efficient prover $P^{*}$ can output a satisfying witness $\omega$ to the statement $\lambda$ (sampled in an interaction with the honest verifier). However, a simulator can commit to $h\left(V^{*}\right)\left(\right.$ instead of $\left.0^{n}\right)$ so that it will have a valid witness to the resulting transcript $\lambda$.

Coming back to our protocol, we use this idea as follows. We modify our first idea, and require the verifier to send a the obfuscation of a new program $M_{\lambda, s}$ (instead of $M_{x, s}$ ) where $\lambda=\langle h, c, r\rangle$ is the transcript of GenStat. The new program $M_{\lambda, s}$ outputs $s$ if and only if it receives a valid witness $\omega$ to the statement $\lambda$ (as described earlier) and $0^{n}$ on all other inputs. To prove the statement $x$ will be proven by proving the knowledge of either a witness $w$ to $x$ or the secret $s$ (using an ordinary witness-indistinguishable proof-of-knowledge (WIPOK)). A simulator can "succeed" in the simulation as before: it commits to verifier's program in $c$ to obtain (an indistinguishable statement) $\lambda$, then uses the fake witness $\omega$ (which it now has) to execute the program $\widetilde{M}_{\lambda, s}(\omega)$ and learn $s$ and complete the WIPOK using $s$.

We now draw attention to some important points arising due to the use of $\lambda$ in the obfuscation (instead of $x$ ). First, the length of the fake witness $\omega$ that the simulator has depends on the length of the program of $V^{*}$. Since the protocol needs to take into account $V^{*}$ of every polynomial length, the obfuscated program $\widetilde{M}_{\lambda, s}$ must accept inputs $\omega$ of arbitrary, a-priori unknown, polynomial length. In other words, the obfuscated program $\widetilde{M}_{\lambda, s}$ must be a Turing machine which accepts inputs of arbitrary, a-priori unknown, (polynomial) length. Therefore, we will have to use program obfuscation for Turing machines.

Second, the statement $\lambda=\langle h, c, r\rangle$ is not a "false" statement since an all powerful prover can always find collisions in $h$ and obtain a satisfying input to $M_{\lambda, s}$. The only guarantee we have is that if $\lambda$ is sampled as above, then it would be hard for any efficient prover - even those with a valid witness to $x$ - to find a satisfying input for $M_{\lambda, s}$. Therefore, unlike before (when $x$ was used instead of $\lambda$ ), obfuscations $\widetilde{M}_{\lambda, s}$ and $\widetilde{M}_{\lambda, 0^{n}}$ are not guaranteed to be indistinguishable if we use an iO-secure obfuscation; this is because the Turing machines $M_{\lambda, s}$ and $M_{\lambda, 0^{n}}$ are not functionally equivalent. Therefore, we will have to use diO-secure obfuscation (since finding a differing input is still hard for these programs). The security of diO is a subtle issue and we discuss it shortly.

By putting these ideas together, we actually a get a standalone ZK protocol for NP (summarized below). The protocol needs to use some kind of reference to $s$ other than the obfuscated program. This is done by using a $f(s)$ where $f$ is a one-way function. This protocol has a "straight line" simulator. Further, unlike Barak's protocol, this protocol does not use universal arguments (and hence the PCP theorem). 
Standalone Zero-Knowledge using Obfuscation. The protocol has three stages.

1. Stage- 1 is the 3 round preamble GenStat: $V$ sends a CRHF $h, P$ sends a commitment $c=\operatorname{Com}\left(0^{n} ; u\right)$ and $V$ sends a random $r \leftarrow\{0,1\}^{n}$.

2. In stage $2, V$ sends $\left(f, \widetilde{s}, \widetilde{M}_{\lambda, s}\right)$ where $f$ is a one-way function, $\widetilde{s}=f(s)$, and $\widetilde{M}_{\lambda, s}$ is the obfuscation of Turing machine $M_{\lambda, s}$ described earlier and $\lambda=\langle h, c, r\rangle$ is the transcript of stage- $1 . V$ also proves that $\left(f, \widetilde{s}, \widetilde{M}_{\lambda, s}\right)$ are correctly constructed (using a standard ZK proof).

3. In stage-3 $P$ provess, using a standard WIPOK, the knowledge of "either a witness $w$ to $x$ or secret $s$ such that $\widetilde{s}=f(s) . "$

Standalone ZK of this protocol can be proven by following Barak's simulator which commits to the code of $V^{*}$ and therefore has an $\omega$ for simulated statement $\lambda$ such that $\widetilde{M}_{\lambda, s}(\omega)=s$ within a polynomial number of steps; the simulator computes $s$ and uses it in the WIPOK. The soundness of the protocol relies on the diO-security of obfuscation. Indeed, following Bar01, for a properly sampled $\lambda$, it is hard to find $\omega$ such that $M_{\lambda, s}(\omega) \neq M_{\lambda, 0^{n}}(\omega)$, and therefore it is hard to distinguish $\widetilde{M}_{\lambda, s}$ from $\widetilde{M}_{\lambda, 0^{n}}$ by diO-security of obfuscation. Now, soundness is argued using three hybrid experiments: first use the simulator of the ZK protocol in stage 2 , then replace $\widetilde{M}_{\lambda, s}$ from $\widetilde{M}_{\lambda, 0^{n}}$, and finally extract $s$ from the WIPOK in stage 3 and violate the hardness of one-way function $f$ (since $x$ is false, extraction must yield $s$ ).

In Section 4, we will discuss why the simulator of this protocol fails in the concurrent setting, and new ideas will be needed to obtain a concurrent ZK protocol. In particular, we will make use of the DGS-oracle idea [DGS09].

Security of diO and the issue of auxiliary information. Continuing from our discussion above, it is clear that for our approach to work obfuscations $\widetilde{M}_{\lambda, s}$ and $\widetilde{M}_{\lambda, 0^{n}}$ should be indistinguishable to a cheating $P^{*}$. However, this is not all: in addition to one of these programs, $P^{*}$ also has access to the statement $\lambda$, which is auxiliary information about the two programs. Therefore, our approach works if we have diO secure w.r.t. auxiliary information (distributed according to $\lambda$ ).

Recent implausibility results of Garg et al. GGHW14 cast serious doubts about the existence of diO w.r.t. arbitrary auxiliary information. While their result does not rule out the possibility of diO w.r.t. specific distributions, we should be extra careful to not rely on auxiliary information which keeps some "secret" such as an obfuscated code [GGHW14].

In our approach, the distribution of $\lambda$ does not have to keep any secrets. In the language of [IPS15, this is public-coin auxiliary information. We show that our approach indeed works by only assuming that the obfuscation is public-coin differing-input secure.

\subsection{Related Work}

Concurrent zero-knowledge. From early on, it was understood and explicitly proven in FS90, GK96, that zero-knowledge is not preserved under parallel 
repetition where multiple sessions of the protocol run at the same time. The more complex notion of concurrent zero-knowledge (cZK) was introduced and achieved by Dwork, Naor, and Sahai DNS98 (assuming "timing constraints" on the underlying network). A large body of research on cZK studied the roundcomplexity of black-box concurrent ZK with improving lower bounds on the same KPR98, Ros00, CKPR03]. The state of art is the lower-bound is by Canetti, Kilian, Petrank, and Rosen CKPR03 who prove that black-box cZK requires at least $O(\log n / \log \log n)$ rounds where $n$ is the length of the statements being proven. Prabhakaran, Rosen, and Sahai [PRS02], building upon the prior works of Richardson and Kilian [RK99] and Kilian and Petrank KP01, presented a cZK protocol for NP which has $\widetilde{O}(\log n)$ rounds, matching the lower bound of CKPR03.

The central open question in this area is to construct a constant round cZK protocol for NP languages based on standard (or at least reasonable) assumptions. Barak Bar01] showed that in the bounded concurrent setting where there is an a-priori upper bound on the number of sessions, there exists a constant round non-black-box cZK protocol for NP; the protocol is based on the existence of collision-resistant hash functions Bar01 and uses universal arguments Kil92, Mic94, Kil95, BG02. The communication complexity of Barak's protocol depends on the a-priori bound on the sessions.

It has proven difficult to extend Barak's NBB techniques to the setting of fully concurrent ZK (i.e., to unbounded polynomially many sessions) in $o(\log n)$ rounds. Nevertheless, NBB techniques have enjoyed great success resulting in the construction of resettable protocols BGGL01, DL07, DGS09, GM11, non-malleable protocols Bar02, PR05b, PR05a], leakage-resilient ZK [Pan14], bounded-concurrent secure computation PR03, Pas04, adaptive security GS12a], and so on. Bitanksy and Paneth BP12a] showed that it is possible to perform non-black-box simulation using oblivious transfer (instead of collision-resistant hash functions and universal arguments). This eventually led to the construction of resettablly-sound ZK under one-way functions BP13a, CPS13, COPV13. Goyal Goy13 presents a non-black-box simulation technique in the fully concurrent setting and achieves the first public-coin cZK protocol in the plain model 4

An alternative approach to construct round-efficient zero-knowledge proofs is to use "knowledge assumptions" Dam91, HT99, BP04. The recent work of Gupta and Sahai GS12b] shows that such assumptions also yield a constant round concurrent ZK protocol for NP. However, all known ZK protocols based on knowledge-type assumptions do not yield an explicit simulator. This is because the knowledge-type assumptions assume the existence of a special "extractor" machine (which is not explicitly known); this extractor is used by the simulator of ZK protocols and only provides an "existential" result.

Chung, Lin, and Pass [CLP13b] recently presented the first construction of a constant-round fully concurrent ZK protocol which has an explicit simulator.

${ }^{4}$ The protocol requires poly $(n)$ rounds. Canetti et al. CLP13a obtain a similar result, albeit in the "global hash" model where a global hash function-which the simulator cannot program - is known to all parties. 
Their result is based on a new complexity-theoretic assumption, namely the existence of so called "strong P-certificates."

Another alternative proposed in the literature is to assume some kind of a setup such as timing constraints, (untrusted) public-key infrastructure, and so on DNS98, DS98, CGGM00, Dam00, Gol02, PTV10, GJO ${ }^{+} 13$, or switch to superpolynomial time simulation Pas03, PV08. We will not consider such models further in this work.

Program obfuscation. After the strong impossibility results of [BGI $\left.{ }^{+} 01\right]$, research in program obfuscation proceeded in two main directions. The first line of research focussed on constructing obfuscation for specific functionalities such as point functions and their variants, proxy re-encryption, encrypted signatures, hyperplanes, conjunctions, and so on Wee05, LPS04, HRSV07, Had10, CRV10, BR13a. The other line of research focussed on finding weaker definitions and alternative models. Goldwasser and Rothblum GR07 considered the notion of best possible obfuscation (and is equivalent to $\mathrm{iO}$ when the obfusactor is polynomial time); and Bitansky and Canetti [BC10] considered virtual grey box security. Alternative models for obfuscation such as the hardware model were considered in $\mathrm{GIS}^{+} 10, \mathrm{BCG}^{+} 11$.

After $\left.\mathrm{GGH}^{+} 13\right]$, an improved construction of $\mathrm{iO}$ was presented by Barak et. al. BGK $^{+13}$. Further, in an idealized "generic encodings" model it is shown that VBB-obfuscation for all circuits can be achieved CV13, BR13b, BGK ${ }^{+} 13$. These results often involve a "bootstrapping step"; Applebaum App13 presents an improved technique for bootstrapping obfuscation. Further complexity-theoretic results appear in recent works of Moran and Rosen [MR13, and Barak et. al. $\mathrm{BBC}^{+} 14$.

Sahai and Waters [SW13] show that indistinguishability obfuscation is a powerful tool and use it to successfully construct several (old and new) cryptographic primitives; further applications of iO appear in [HSW13, BZ13, BCP14, KRW13, MO13

Differing input obfuscation was studied by Ananth et. al. $\left[\mathrm{ABG}^{+} 13\right.$, who present a candidate construction of $\mathrm{diO}$ for the class of polynomial time Turing machines and demonstrate new applications. Another variant of their construction allows the Turing machines to accept variable length inputs. Concurrent work of Boyle, Chung, and Pass BCP14 introduces a related notion of extractability obfuscation and shows this notion (and diO) are implied by $\mathrm{iO}$ when the programs differ only on polynomially many inputs. In addition, it also presents obfuscation for the class of polynomial time Turing machines, building upon the work of Brakerski and Rothblum BR13a. Very recently, in concurrent and independent works, construction for bounded-space RAM programs were presented by relying on iO and OWFs BGT14] and other additional assumptions [CHJV14, [LP14].

The issue of auxiliary information in program obfuscation was first considered by Goldwasser and Kalai GK05, and further explored in GK13, BCPR13, BP13b, GGHW14, IPS15. The work of Bitansky, Canetti, Paneth, and Rosen BCPR13. shows that if iO exists then "extractability primitives" such as knowledge-types assumptions and extractable one-way functions [CD09] cannot 
exist in the presence of arbitrary auxiliary information. Boyle and Pass BP13b strengthen this result further by showing a pair of (universal) distributions $\mathcal{Z}, \mathcal{Z}$, on auxiliary information such that either extractable OWF w.r.t. $\mathcal{Z}$ do not exist or extractability-obfuscations w.r.t. $\mathcal{Z}$ ' do not exist. The work of Garg, Gentry, Halevi, and Wichs GGHW14 shows that diO w.r.t. arbitrary auxiliary information cannot exist if certain specific obfuscation assumption is true. Ishai, Pandey, and Sahai IPS15] formulate the notion of public-coin diO in which the auxiliary is restricted to be merely the random coins of the sampler, and recover much of the existing applications of diO under this new notion.

\section{Preliminaries}

We use standard notations which are recalled here. This section can be skipped without affecting readability.

Notation. For a randomized algorithm $A$ we write $A(x ; r)$ the process of evaluating $A$ on input $x$ with random coins $r$. We write $A(x)$ the process of sampling a uniform $r$ and then evaluating $A(x ; r)$. We define $A(x, y ; r)$ and $A(x, y)$ analogously. We denote by $\mathbb{N}$ and $\mathbb{R}$ the set of natural and real numbers respectively. The concatenation of two string $a$ and $b$ is denoted by $a \| b$.

We assume familiarity with interactive Turing machines (ITMs). For two randomized ITMs $A$ and $B$, we denote by $[A(x, y) \leftrightarrow B(x, z)]$ the interactive computation between $A$ and $B$, with $A$ 's inputs $(x, y)$ and $B$ 's inputs $(x, z)$, and uniform randomness; and $\left[A\left(x, y ; r_{A}\right) \leftrightarrow B\left(x, z ; r_{B}\right)\right]$ when we wish to specify randomness. We denote by $\operatorname{VIEW}_{P}[A(x, y) \leftrightarrow B(x, z)]$ and $\mathrm{OUT}_{P}[A(x, y) \leftrightarrow$ $B(x, z)]$ the view and output of machine $P \in\{A, B\}$ in this computation. Finally, TRANS $[A(x, y) \leftrightarrow B(x, z)]$ denotes the transcript of the interaction $[A(x, y) \leftrightarrow B(x, z)]$ which consists of all messages exchanged in the computation.

We also assume familiarity with oracle Turing machines, which are ordinary TMs with an extra tape called the oracle communication tape. An oracle TMs $A$ will be written as $A^{\langle\cdot\rangle}$ to insist that it is an oracle TM; in addition, we write $A^{\mathcal{I}}$ when $A$ 's oracle is fixed to $\mathcal{I}$. Recall that each query to $\mathcal{I}$ counts as one step towards the running time of $A^{\mathcal{I}}$.

Unless specified otherwise, all algorithms receive a parameter $n \in \mathbb{N}$, called the security parameter, as their first input. Often, the security parameter will not be mentioned explicitly and dropped from the notation. With some exceptions, all algorithms run in poly $(n)$ steps and all inputs have poly $(n)$ length. A function negl : $\mathbb{N} \rightarrow \mathbb{R}$ is negligible if it approaches zero faster than every polynomial.

Two ensembles $\{\mathcal{X}\}_{n \in \mathbb{N}}$ and $\left\{\mathcal{Y}_{\backslash}\right\}_{n \in \mathbb{N}}$ are said to be computationally indistinguishable, denoted $\{\mathcal{X}\} \stackrel{c}{\approx}\{\mathcal{Y} \backslash\}$, if for all non-uniform probabilistic polynomial time (PPT) distinguishers $D$, sufficiently large $n$, and every advice string $z_{n}$ : $\left|\operatorname{Pr}_{x \leftarrow \mathcal{X}_{n}}\left[D_{n}(x)=1\right]-\operatorname{Pr}_{y \leftarrow \mathcal{Y}_{n}}\left[D_{n}(y)=1\right]\right| \leq \operatorname{negl}(n)$, where we write $D_{n}(a)$ to denoted $D\left(n, z_{n}, a\right)$, and negl is a negligible function. The statistical distance between two probability distributions $\mathcal{X}$ and $\mathcal{Y}$ over the same support $S$ is 
denoted by $\Delta(X, Y)=\frac{1}{2} \sum_{a \in S}|\operatorname{Pr}[X=a]-\operatorname{Pr}[Y=a]|$. We say that ensembles $\left\{\mathcal{X}_{n}\right\}_{n \in \mathbb{N}}$ and $\left\{\mathcal{Y}_{n}\right\}_{n \in \mathbb{N}}$ are statistically indistinguishable (or statistically close), denoted $\left\{\mathcal{X}_{n}\right\} \stackrel{s}{\approx}\left\{\mathcal{Y}_{n}\right\}$, if there exists a negligible function negl such that $\Delta\left(\mathcal{X}_{n}, \mathcal{Y}_{n}\right) \leq \operatorname{negl}(n)$ for all sufficiently large $n$.

Standard primitives. In this work, we will be using a family of injective one-way functions. In addition, unless specified otherwise, we assume that all functions $f \in \mathcal{F}_{n}$ in the family have an efficiently testable range membership: i.e., there exists a polynomial time algorithm to test that $y \in \operatorname{Range}(f)$ where $\operatorname{Range}(f)$ denotes the range of $f$.

We will also be using a family of collision resistant hash functions (CRHF) $\left\{\mathcal{H}_{n}\right\}$ where $h:\{0,1\}^{*} \rightarrow\{0,1\}^{\text {poly }(n)}$ for $h \in \mathcal{H}_{n}$; recall that $\left\{\mathcal{H}_{n}\right\}$ is a CRHF family if there exists a negligible function negl such that for every nonuniform PPT machines $A$, every sufficiently large $n$, and every advice string $z_{n}$ : $\operatorname{Pr}_{h \leftarrow \mathcal{H}_{n}}\left[h(x)=h(y):(x, y) \leftarrow A\left(z_{n}, h\right)\right] \leq \operatorname{negl}(n)$.

Finally, we will also be using a non-interactive, perfectly binding commitment scheme for committing strings of polynomial length. A commitment to a string $m$ using randomness $u$ will be denoted by $c=\operatorname{Com}(m ; u)$. Without loss of generality, we assume that the message $m$ committed to in $c$ can be recovered given the randomness $u$ and the string $c$. We assume perfectly binding schemes purely for the simplicity of exposition. One can replace Com by the 2-round statistically-binding commitment scheme of Naor Nao89. without affecting our results.

\subsection{Interactive Proofs, Proofs of Knowledge, and Witness Indistinguishability}

We recall the standard definitions of interactive proofs GMR85, witness indistinguishability [FS90, and proofs of knowledge GMR85, TW87, FFS88, FS90, BG92, PR05b.

Definition 1 (Interactive Proofs). A pair of probabilistic polynomial time interactive Turing machines $\langle P, V\rangle$ is called an interactive argument system for a language $\mathbf{L} \in \mathbf{N P}$ with witness relation $\mathbf{R}$ if there exists a negligible function negl $: \mathbb{N} \rightarrow \mathbb{R}$ such that the following two conditions hold:

- Completeness: for every $x \in \mathbf{L}$, and every witness $w$ such that $\mathbf{R}(x, w)=1$, it holds that

$$
\operatorname{Pr}\left[\mathrm{OUT}_{V}[P(x, w) \leftrightarrow V(x)]=1\right]=1 .
$$

- Soundness: for every $x \notin \mathbf{L}$, every interactive Turing machine $P^{*}$ running in time at most poly $(|x|)$, and every $y \in\{0,1\}^{*}$,

$$
\operatorname{Pr}\left[\mathrm{OUT}_{V}\left[P^{*}(x, y) \leftrightarrow V(x)\right]=1\right] \leq \operatorname{negl}(|x|) .
$$

If the soundness condition holds for every (not necessarily PPT) machine $P^{*}$ then $\langle P, V\rangle$ is called an interactive proof system. 
The probability in the soundness condition is called the soundness error of the system, and we say that the system has negligible soundness error since this probability is at most negl $(|x|)$. Although, traditionally soundness error is defined in terms of the statement length $|x|$, in cryptographic contexts, it is convenient to define it in terms of the security parameter $n$, and write $\operatorname{negl}(n)$. This is without loss of generality, since in our setting since $|x|=\operatorname{poly}(n)$. Also, in this work, we will use words "argument" and "proof" interchangeably throughout the paper.

Definition 2 (Proof of Knowledge). Let $\langle P, V\rangle$ be an interactive proof system for a language $\mathbf{L} \in \mathbf{N P}$ with witness relation $\mathbf{R}$. We say that $\langle P, V\rangle$ is a proof of knowledge (POK) for relation $\mathbf{R}$ if there exists a polynomial $p$ and a probabilistic oracle machine $E$ (called the extractor) such that for every PPT ITM $P^{*}$, there exists a negligible function negl such that for every $x \in \mathbf{L}$, and every $(y, r) \in\{0,1\}^{*}$ such that $q_{x, y, r}:=\operatorname{Pr}\left[\mathrm{OUT}_{V}\left[P_{x, y, r}^{*} \leftrightarrow V(x)\right]=1\right]>0$ where $P_{x, y, r}^{*}$ denotes the machine $P^{*}$ whose common input, auxiliary input, and randomness are fixed to $x, y$ and $r$ respectively and the probability is taken over the randomness of $V$, the following conditions holds:

- the expected number of steps taken by $E^{P_{x, y, r}^{*}}$ is bounded by $\frac{p(|x|)}{q_{x, y, r}}$, where $E^{P_{x, y, r}^{*}}$ is machine $E$ with oracle access to $P_{x, y, r}^{*}$;

- except with negligible probability, $E^{P_{x, y, r}^{*}}$ outputs $w^{*}$ such that $\mathbf{R}\left(x, w^{*}\right)=1$.

Definition 3 (Witness Indistinguishable Proofs). Let $\langle P, V\rangle$ be an interactive proof system for a language $\mathbf{L} \in \mathbf{N P}$ with witness relation $\mathbf{R}$. We say that $\langle P, V\rangle$ is witness indistinguishable (WI) for relation $\mathbf{R}$ if for every PPT ITM $V^{*}$, every statement $x \in \mathbf{L}$, every pair of witnesses $\left(w_{1}, w_{2}\right)$ such that $\mathbf{R}\left(x, w_{i}\right)=1$ for every $i \in\{1,2\}$, and every (advice) string $z \in\{0,1\}^{*}$, it holds that $\left\{\mathrm{VIEW}_{|x|}^{(1)}\right\} \stackrel{c}{\approx}\left\{\operatorname{VIEW}_{|x|}^{(2)}\right\}$ where $\left\{\operatorname{VIEW}_{|x|}^{(i)}\right\}:=\operatorname{VIEW}_{V^{*}}\left[P\left(x, w_{i}\right) \leftrightarrow V^{*}(x, z)\right]$.

As before, w.l.o.g., we can replace $|x|$ by the security parameter $n$ in all definitions above. We remark that there exists a WIPOK with strict polynomial time extraction in constant rounds using non-black-box techniques BL04] and in $\omega(1)$ rounds using black-box techniques GMR85, Blu87.

Three round, public-coin WIPOKand ZAPs. The classical protocols of GMR85, Blu87, based on the existence of non-interactive perfectly binding commitment schemes, are 3-round witness indistinguishable, proof of knowledge (WIPOK) protocols (for every language in NP). We will use Blum's protocol [Blu87] as a building block and denote its three messages by $\langle\alpha, \beta, \gamma\rangle$, where $\beta$ is random string of sufficient length 5

\footnotetext{
${ }^{5}$ We remark that this protocol has a black-box extractor whose expected running time is proportional to the inverse of a cheating prover's success probability. However, there also exist WIPOK with strict polynomial time extraction in constant rounds using non-black-box techniques [BL04] and in $\omega(1)$ rounds using black-box techniques GMR85, Blu87.
} 
A ZAP for a language $\mathbf{L}$, introduced by Dwork and Naor DN00, is a two round witness indistinguishable interactive proof for $\mathbf{L}$. ZAPs can be constructed from a variety of assumptions such as non-interactive zero-knowledge proofs BFM88, BSMP91 (which in turn can be based on trapdoor permutations [FLS99]) and verifiable random functions MRV99. In fact, even non-interarctive (i.e., one round) constructions for ZAPs for all of NP exist based on bilinear pairings GOS06 and derandomization techniques BOV03.

We will use the two round construction of [DN00] based on NIZK as a building block and denote its two messages by $\langle\sigma, \pi\rangle$ where $\sigma$ is a randomly string of sufficient length. An important property of this construction is adaptive soundness: the statement to be proven can be chosen after the string $\sigma$ has been sent by the verifier. We will rely on this property in our security proofs.

\subsection{Concurrent Zero Knowledge}

We now recall the notion of concurrent zero-knowledge DNS98] in which one considers a "concurrent adversary" $V^{*}$ who interacts in many copies of $P$, proving adaptively chosen, possibly correlated, polynomially many statements. We follow conventions established in [DNS98, PRS02, Ros04].

Concurrent attack. The concurrent attack on an interactive proof systems $\langle P, V\rangle$ for language $\mathbf{L} \in \mathbf{N P}$ with witness relation $\mathbf{R}$ considers an arbitrary interactive TM $V^{*}$ which opens at most $m=m(n)$ sessions for an arbitrary polynomial $m$ with arbitrary auxiliary input $z \in\{0,1\}^{*}$. Let $\boldsymbol{x}:=\left\{x_{i}\right\} \in \mathbf{L}^{m}$ be set of statements in $\mathbf{L}$ of length at most $\operatorname{poly}(n)$, and $\boldsymbol{w}:=\left\{w_{i}\right\}_{i \in[m]}$ be such that $\mathbf{R}\left(x_{i}, w_{i}\right)=1$. The attack proceeds by uniformly fixing the random coins of $V^{*}$ and initiating its execution on input the security parameter $n \in \mathbb{N}$ and auxiliary input $z$. At each step, $V^{*}$ either initiates a new session - in which case a new prover instance $P\left(x_{i}, w_{i}\right)$ with fresh randomness is fixed who interacts with $V^{*}$ in session $i$; or $V^{*}$ schedules the delivery of a message of an existing session in which the corresponding prover instance responds with corresponding message. There is no restriction on how $V^{*}$ schedules the messages of various sessions. We say that $V^{*}$ launches $m$-concurrent attack on $\langle P, V\rangle$. The output of the attack consists of the view of $V^{*}$, denoted $\operatorname{VIEW}_{V^{*}}^{\langle P, V\rangle}(n, m, \boldsymbol{x}, \boldsymbol{w}, z)$.

Definition 4 (Concurrent Zero Knowledge). We say that an interactive proof system $\langle P, V\rangle$ for a language $\mathbf{L} \in \mathbf{N P}$ (with witness relation $\mathbf{R}$ ) is concurrent zero knowledge if for every polynomial $m: \mathbb{N} \rightarrow \mathbb{N}$, every PPT ITM $V^{*}$ launching a m-concurrent attack, there exists a PPT machine $S_{V^{*}}$ such that for every set $\boldsymbol{x}:=\left\{x_{i}\right\} \in \mathbf{L}^{m}$ of statements of length at most poly $(n)$, every $\boldsymbol{w}:=\left\{w_{i}\right\}_{i \in[m]}$ such that $\mathbf{R}\left(x_{i}, w_{i}\right)=1$, and every auxiliary input $z \in\{0,1\}^{*}$ it holds that

$$
\left\{S_{V^{*}}(n, \boldsymbol{x}, z)\right\}_{n \in \mathbb{N}} \stackrel{c}{\approx}\left\{\operatorname{VIEW}_{V^{*}}^{\langle P, V\rangle}(n, m, \boldsymbol{x}, \boldsymbol{w}, z)\right\}_{n \in \mathbb{N}} .
$$

Machine $S_{V^{*}}$ is called the simulator. 
In what follows, we will sometimes abuse the notation and write $V^{*}$ to also mean the description of the Turing machine $V^{*}$. However, when we want to be explicit about the description of a Turing machine $M$ (including $V^{*}$ ), we will actually write $\operatorname{desc}(M)$. For the simulator, we may sometimes write $S_{V^{*}}(\cdot):=S\left(V^{*}, \cdot\right)$ to insist that the program of $V^{*}$ is given as an explicit input to the simulator (and drop $n$ from the notation). Further, we will assume a (unique) session identifier for each session represented by a string of length $n$; this session identifier can be chosen by $V^{*}$ so long as it is unique for every session. W.l.o.g. we assume that the all-ones string $1^{n}$ (not to be confused with the unary representation of the security parameter) is never used as a session identifier and denotes a special symbol.

\section{Differing Input Obfuscation for Turing Machines}

In this section, we recall the notion of public-coin differing input obfuscation (pcdiO) for Turing machines IPS15. Let Steps $(M, x)$ denote the number of steps taken by a TM $M$ on input $x$; we use the convention that if $M$ does not halt on $x$ then $\operatorname{Steps}(M, x)$ is defined to be the special symbol $\infty$. Let $\mathcal{M}=\left\{\mathcal{M}_{n}\right\}$ denote a parameterized collection of polynomial size and polynomial time TMs, i.e., there exists a global polynomial $a$ such that for every $n \in \mathbb{N}$, every $M \in \mathcal{M}_{n}$, $|M| \leq a(n)$ and $\operatorname{Steps}(M, x) \leq a(|x|)$ where $x$ can be of arbitrary polynomial length.

We say that a pair of TMs $\left(M_{0}, M_{1}\right)$ in the class $\mathcal{M}_{n}$ (for any $n$ ) is compatible if they have the same size and for every $x, \operatorname{Steps}\left(M_{0}, x\right)=\operatorname{Steps}\left(M_{1}, x\right)$.

Definition 5 (Compatible TMs). A pair of Turing machines $\left(M_{0}, M_{1}\right) \in$ $\mathcal{M}_{n} \times \mathcal{M}_{n}$ for $n \in \mathbb{N}$ is said to be compatible if $\left|M_{0}\right|=\left|M_{1}\right|$ and for every string $x \in\{0,1\}^{*}$ it holds that $\operatorname{Steps}\left(M_{0}, x\right)=\operatorname{Steps}\left(M_{1}, x\right)$.

We remark that the notion of compatible TMs allows the obfuscation to leak the running time of the obfuscated TMs. This is standard requirement; we can also use the convention of $\mathrm{ABG}^{+} 13$. IPS15] where the TMs also output their running time in addition to the "official" output.

We now recall the notion of public-coin differing inputs sampler IPS15. Roughly speaking, Samp is public-coin differing-inputs sampler if, on input the random coins $z$, it output a pair of compatible TMs $\left(M_{0}, M_{1}\right)$ such that no PPT adversary $A$ having access to $z$ can produce an $x$ such that: $M_{0}(x) \neq M_{1}(x)$. We use a slightly different notation form [IPS15, and require that in addition to outputting $M_{0}, M_{1}$, Samp also outputs its random coins. The randomness $z$ of Samp will then not be mentioned as an explicit input. The definition follows.

Definition 6 (Public-coin Differing-Inputs Sampler for TMs). We say that a (possibly non-uniform) PPT Turing machine Samp is a public-coin differinginputs sampler for Turing machines if the following conditions hold:

1. the output of $\operatorname{Samp}\left(1^{n}\right)$ is a triplet $\left(z, M_{0}, M_{1}\right)$ such that $z$ is the randomness of Samp and $\left(M_{0}, M_{1}\right) \in \mathcal{M}_{n} \times \mathcal{M}_{n}$ is always a pair of compatible TM $s$; 
2. for every (possibly non-uniform) PPT TM $A$ there exists a negligible function negl such that for all $n \in \mathbb{N}$ :

$$
\operatorname{Pr}\left[M_{0}(x) \neq M_{1}(x):\left(z, M_{0}, M_{1}\right) \leftarrow \operatorname{Samp}\left(1^{n} ; z\right) ; A\left(z, M_{0}, M_{1}\right)=x\right] \leq \operatorname{negl}(n) .
$$

For convenience, a public-coin differing-input sampler will also be referred to as $a$ nice sampler.

Public-coin differing-input obfuscator. We now present the definition of a publiccoin differing input obfuscator for Turing machines. Roughly speaking, the notion states that a machine $\mathcal{O}$ is a pc-diO if the following holds: if there exists a PPT distinguisher $D$ who distinguishes $\mathcal{O}\left(M_{0}\right)$ from $\mathcal{O}\left(M_{1}\right)$ when given as auxiliary input the random coins $z$ of the sampler who samples $\left(M_{0}, M_{1}\right)$, then it is easy to find an $x$ (given $z$ ) such that $M_{0}(x) \neq M_{1}(x)$. In other words, if it is hard to find the "differing input" $x$ then the two obfuscations are indistinguishable.

Definition 7 (Public-coin Differing-Inputs Obfuscator for Turing Machines, [IPS15]). A uniform PPT machine $\mathcal{O}$ is called a public-coin differing input obfuscator (pc-diO) for a class of Turing machines $\left\{\mathcal{M}_{n}\right\}$ if the following conditions are satisfied:

1. Polynomial slowdown and functionality: there exists a polynomial $a_{\mathrm{dio}}$ such that for every $n \in \mathbb{N}$, every $M \in \mathcal{M}_{n}$, every input $x$, and every $\widetilde{M} \leftarrow$ $\mathcal{O}(n, M)$, the following conditions hold:

$$
\begin{aligned}
& -\operatorname{Steps}(\widetilde{M}, x) \leq a_{\text {dio }}(n, \operatorname{Steps}(M, x)) \\
& -\widetilde{M}(x)=M(x)
\end{aligned}
$$

Polynomial $a_{\text {dio }}$ is called the slowdown polynomial of $\mathcal{O}$.

2. Indistinguishability: for every public-coin differing-input (a.k.a. nice) sampler Samp (i.e., satisfying definition [6), for every (possibly non-uniform) PPT distinguisher D, there exists a negligible function negl such that for all $n$ :

$$
\begin{aligned}
& \mid \operatorname{Pr}\left[D\left(z, \mathcal{O}\left(n, M_{0}\right)\right)=1:\left(z, M_{0}, M_{1}\right) \leftarrow \operatorname{Samp}\left(1^{n} ; z\right)\right] \\
& -\operatorname{Pr}\left[D\left(z, \mathcal{O}\left(n, M_{1}\right)\right)=1:\left(z, M_{0}, M_{1}\right) \leftarrow \operatorname{Samp}\left(1^{n} ; z\right)\right] \mid \leq \operatorname{negl}(n) .
\end{aligned}
$$

where the probability is taken over the randomness of both Samp and $\mathcal{O}$.

Candidate constructions. In [PS15, a candidate construction of pc-diO for all polynomial time TMs with variable length input of polynomial size is presented. The functionality of the construction allows the TMs to accept inputs of any length, even larger than polynomial. The security states that if a PPT machine distinguishes the obfuscation of the given TMs, there will exist an input of polynomial size, which can be extracted, such that the two machines will differ on that input. The assumptions underlying this construction are: pc-diO for $N C^{1}$ circuits, fully homomorphic encryption, and SNARKs for NP. The construction 
of $\mathrm{GGH}^{+} 13$ is seen as a plausible candidate for pc-diO for $N C^{1}$ and existing implausibility results GGHW14 are unlikely to have a consequence to this assumption. Construction of diO with stronger forms of auxiliary input - worst case in which the security must hold for all auxiliary strings $\mu$, and distributional where it holds for specific distributions of $\mu$-were presented in $\left[\mathrm{ABG}^{+} 13, \overline{\mathrm{BCP}} 14\right.$.

\section{Constant Round Concurrent Zero-knowledge}

The simplest way to see why the protocol of previous section does not work in the concurrent setting is to consider its execution in a recursively interleaved schedule (described by Dwork, Naor, and Sahai [DNS98). In the context of our protocol, this schedule will have $n$ sessions interleaved recursively as follows: session $n$ does not "contain" any messages of any other session, and all messages of session $i$ are contained between messages $c_{i-1}$ and $r_{i-1}$ of session $i-1$ for every $i$, starting from $i=n$. A pictorial representation of this scheduling is given in the full version PPS15. The double-headed arrows marked by $\pi_{i}$ represent the rest of the messages of the $i$-th session. Roughly speaking, the simulation fails because of the following: in order to simulate session $i$, the simulator needs to extract the secret $s_{i}$ by running the program $\widetilde{M}_{\lambda_{i}, s_{i}}$; however, the execution of $\widetilde{M}_{\lambda_{i}, s_{i}}$ contains an execution of $\widetilde{M}_{\lambda_{i+1}, s_{i+1}}$ and due to this recursion, simulator's total running time in session 1 is exponential in $n$.

Formally, let $t_{3} \geq 1$ be the time taken by the verifier in computing $r_{3}$ on input the string $c_{3}$. Then clearly, the time taken by the simulator in running the obfuscated machine $\widetilde{M}_{\lambda_{3}, s_{3}}$ is $T_{3} \geq t_{3}$. Then, if $t_{2}$ denotes the time taken by the simulator to obtain string $r_{2}$, we have that $t_{2} \geq t_{3}+T_{3} \geq 2 t_{3}$. Clearly, the time taken by the simulator to extract $s_{2}$ by running the program $\widetilde{M}_{\lambda_{2}, s_{2}}$ will be at least $T_{2} \geq t_{2} \geq 2 t_{3}$. By repeating this argument for session 1 , we have that $T_{1} \geq t_{1} \geq t_{2}+T_{2} \geq 2 t_{2} \geq 2^{2} t_{3}$. Repeating this argument for $n$ sessions in the DNS schedule, the total time taken by the simulator will be $\geq 2^{n-1}$.

Avoiding recursive computation via DGS-oracle. It is clear that the reason our stand-alone simulator runs in exponential time is because in order to compute $s_{i}$ for session $i$, the simulator runs (the obfuscation of) a program which recursively runs such a program for every interleaved session between $c_{i}$ and $r_{i}$. That is, the program $\widetilde{M}_{\lambda_{i}, s_{i}}$ ends up recomputing all of the secrets of the interleaved sessions even though they have already been computed.

We can avoid this recomputation as follows. Let $\mathcal{I}$ be an oracle which takes as input queries of the form $(f, \widetilde{s})$-where $f$ is an injective one-way function and $\widetilde{s}$ is in the range of $f$-and returns the unique value $s$ such that $f(s)=\widetilde{s} 6$ Now consider an arbitrary program $\Pi^{\mathcal{I}}$ which has access to the inversion oracle $\mathcal{I}$. Clearly, if $r$ is chosen randomly, then for any (fixed) program $\Pi^{\mathcal{I}}$ and any fixed input $a$, the probability that $\Pi^{\mathcal{I}}(a)=r$ is at most $2^{-n}$. This is because

${ }^{6}$ We assume that it is easy to test that $f$ is injective and that $\widetilde{s}$ is in the range of $f$. These requirements are only for simplicity and the protocol works even if it is not easy to test these properties. 
once the description of the oracle program $\Pi^{\langle\cdot\rangle}$ is fixed, the output of $\Pi^{\mathcal{I}}(a)$ is deterministically fixed (for any fixed input $a$ chosen prior to seeing $r$ ) and $r$ hits this value with probability at most $2^{-n}$.

Our main point here is that it is hard to come up with a satisfying "fake witness" $\omega$ to the transcripts $\lambda=\langle h, c, r\rangle$ even if the program committed in $c$ is given access to the inversion oracle $\mathcal{I}$. On the other hand, the simulator can still predict $r$ as before. However, more importantly, by means of the oracle $\mathcal{I}$ we can avoid the recursive re-computation of the secrets in the concurrent setting as follows.

Consider an alternative simulator $S^{\langle\cdot\rangle}$ which will be given access to the oracle $\mathcal{I}$. This simulator will have access to both, the program of the verifier $V^{*}$ as well as its own program, given as explicit inputs, collectively denoted as $\Pi_{S, V^{*}}^{\langle\cdot\rangle}$. The simulator, on input a session index $i$, will work by initiating an execution of $V^{*}$. It will commit to program $\Pi_{S, V^{*}}^{\langle\cdot\rangle}(j)$ in session $j$ (ignoring for the moment the fact that simulator needs fresh randomness); finally, this simulator does not run any obfuscated program to compute the secrets. Instead it queries the oracle $\mathcal{I}$ on "well formed" $\left(f_{j}, \widetilde{s}_{j}\right)$ for every session $j \neq i$; when $j=i$ it simply returns the string $r_{i}$. Then, if all goes well, observe that program $\Pi^{\langle\cdot\rangle}(i)$ predicts string $r_{i}$ in polynomial time (given $\mathcal{I}$ ) and this holds for every session $i$. In particular, there is no recursive recomputation of the secrets since they can be fed to the program directly once they have been computed. We note that such an oracle was first used by Deng, Goyal, and Sahai DGS09] to construct the first resettably-sound resettable zero-knowlege protocol for NP.

It should be clear that the actual simulation will be performed by a "main" simulator $S_{\text {main }}$ which will not have access to any inversion oracle, and run in (strict) polynomial time. The main simulator will run in the same manner as the alternative simulator $S^{\langle\cdot\rangle}$ except that instead of using $\mathcal{I}$, it will run the obfuscated programs (only once for each session) to recover the secrets. To ensure efficient simulation, once a session secret has been recovered, it will be stored in a global table $\mathcal{T}$ (which will be used to simulate answers of $\mathcal{I}$ ). Therefore the "fake witness" will now have the form $\omega=\left\langle u, \Pi^{\langle\cdot\rangle}, \mathcal{T}\right)$, but the statements will still have the same form $\lambda=\langle h, c, r\rangle$; and we require that $\Pi^{\mathcal{T}}$ outputs $r$ within finite steps (see details below).

Relation $\mathbf{R}_{\text {sim }}$ and the simple variant of our protocol. To formally capture the above mentioned requirement for the transcripts $\lambda$, we define a relation $\mathbf{R}_{\text {sim }}$ in figure 1. The family of injective one-way functions is denoted by $\left\{\mathcal{F}_{n}\right\}_{n \in \mathbb{N}}$ and that of collision-resistant hash functions by $\left\{\mathcal{H}_{n}\right\}_{n \in \mathbb{N}}$. An important observation regrading $\mathbf{R}_{\text {sim }}$ is that since table $\mathcal{T}$ is not a part of the commitment $c$ (and it should not be), we must enforce that $\Pi^{\langle\cdot\rangle}$ never makes any invalid queries to $\mathcal{T}$. This is because after seeing $r$, it is easy to design a "bad" table $\mathcal{T}$ which will encode $r$ by means of "bad" entries and "satisy" $\lambda$.

Relation $\mathbf{R}_{\text {sim }}$ allows us to prove that no efficient prover can compute $\omega$ such that $\mathbf{R}_{\operatorname{sim}}(\lambda, \omega)=1$ with noticeable probability where $\lambda$ is the transcript of GenStat with an honest verifier. We prove this claim formally in lemma 1 under the collision-intractability of $\left\{H_{n}\right\}$. We note that $\mathbf{R}_{\text {sim }}$ is not decidable in 
polynomial time in general, but this will not be an issue for our reductions since we will ensure that it is checked only on "good" instances (which can be verified in polynomial time).

Instance: $\quad$ A tuple $\langle h, c, r\rangle \in \mathcal{H}_{n} \times\{0,1\}^{\text {poly }(n)} \times\{0,1\}^{n}$ where $h$ : $\{0,1\}^{*} \rightarrow\{0,1\}^{n}$.

Witness: A tuple $\left\langle u, \Pi^{\langle\cdot\rangle}, \mathcal{T}\right\rangle \in\{0,1\}^{\text {poly }(n)} \times\{0,1\}^{*} \times\{0,1\}^{*}$ where $\Pi^{\langle\cdot\rangle}$ is an oracle Turing machine, and $\mathcal{T}$ is a table containing entries of the form $(f, \widetilde{s}, s)$ such that when queried on $(f, \widetilde{s}), \mathcal{T}$ returns $s$, denoted $\mathcal{T}(f, \widetilde{s})=s$.

Relation: $\quad \mathbf{R}_{\text {sim }}\left(\langle h, c, r\rangle,\left\langle u, \Pi^{\langle\cdot\rangle}, \mathcal{T}\right\rangle\right)=1$ if and only if all of the following conditions hold:

1. $c=\operatorname{Com}\left(h\left(\Pi^{\langle\cdot\rangle}\right) ; u\right)$

2. $\forall(f, \widetilde{s}, s) \in \mathcal{T}$ it holds that $f \in \mathcal{F}_{n}$ is an injective function and $f(s)=\widetilde{s}$

3. Program $\Pi^{\mathcal{T}}$, takes no input, outputs the string $r$, and halts within $2^{n}$ steps.

4. Program $\Pi^{\mathcal{T}}$ makes oracle queries of the form $(f, \widetilde{s})$ such that:

$$
\forall \text { queries }(f, \widetilde{s}) \exists s \text { s.t. }(f, \widetilde{s}, s) \in \mathcal{T}
$$

Fig. 1. Relation $\mathbf{R}_{\text {sim }}$ based on a perfectly binding commitment Com.

We are now ready to describe the simpler variant of our protocol which is constant round and concurrent zero-knowledge. The protocol has three stages: in first stage $\lambda$ is sampled, in second stage the verifier sends $(f, \widetilde{s})$ and Turing machine $\widetilde{M}_{\lambda, s}$ (and also proves that is is a correctly generated), in stage $3, P$ proves the knowledge of either a witness to $x$ (the statement) or $s$ s.t. $f(s)=\widetilde{s}$. The formal description of our protocol, named Simple-c $\mathcal{Z K}$, appears in figure 2 In the protocol description, we have renamed the machine $M_{\lambda, s}$ (which was only informally stated earlier) to $\operatorname{SimLock}_{\lambda, s}(\cdot):=\operatorname{SimLock}(\lambda, \cdot s)^{7}$ where, formally:

$\operatorname{SimLock}(\lambda, \omega, s)$ :

- Test if $\mathbf{R}_{\operatorname{sim}}(\lambda, \omega)=1$, and if so output $s$; else, output $0^{n}$.

Relation $\mathbf{R}_{\text {sim }}^{a}$, language $\mathbf{L}_{\text {sim }}^{a}$. Relation $\mathbf{R}_{\text {sim }}$ is undecidable in polynomial in general. We define a polynomial time decidable version of $\mathbf{R}_{\text {sim }}$. For a polynomial $a: \mathbb{N} \rightarrow \mathbb{N}$, relation $\mathbf{R}_{\text {sim }}^{a}$ is defined as follows: $\mathbf{R}_{\text {sim }}^{a}$ is identical to $\mathbf{R}_{\text {sim }}$ except that the witness $(u, \Pi<\cdot\rangle, \mathcal{T})$ satisfies following additional constraints: $(1)$ $|\mathcal{T}| \leq a(n)$, and $(2) \Pi^{\mathcal{T}}$ halts in at most $a(n)$ steps.

7 SimLock stands for "simulator's lock," i.e., only the simulator will be able to "unlock" the secret $s$ from this program. 
Inputs. The common input to $P$ and $V$ is a statement $x \in \mathbf{L}$ where language $\mathbf{L} \in \mathbf{N P}$. The prover's auxiliary input is a witness $w$ such that $\mathbf{R}(x, w)=1$. The security parameter $n$ is an implicit input to both parties.

Protocol. The protocol proceeds in three stages.

Stage 1: $P$ and $V$ execute the GenStat protocol in which $V$ sends the first message $h \leftarrow \mathcal{H}_{n}, P$ sends the second message $c=\operatorname{Com}\left(0^{n} ; u\right)$ for a random $u$, and $V$ sends the final message $r \leftarrow\{0,1\}^{n}$. Let $\lambda=\langle h, c, r\rangle$ be the transcript.

Stage 2: $V$ samples an injective one-way functions $f \leftarrow \mathcal{F}_{n}$, a random input $s \in\{0,1\}^{n}$, and a sufficiently long random tape $\zeta \in\{0,1\}^{\text {poly }(n)}$ and computes:

$$
\widetilde{s}=f(s), \quad \widetilde{M}_{\lambda, s} \leftarrow \mathcal{O}\left(\operatorname{SimLock}_{\lambda, s} ; \zeta\right)
$$

$V$ sends $\left(f, \widetilde{s}, \widetilde{M}_{\lambda, s}\right)$, and proves using a constant round ZK protocol (say) $\left.\Pi_{\mathrm{ZK}}\right)$ that there exist $(s, \zeta)$ satisfying equation (1) above.

Stage 3: $P$ proves to $V$, using a 3 -round WIPOK (say $\Pi_{\text {WIPOK }}$ ) the knowledge of either:

- $w$ such that $\mathbf{R}(x, w)=1$; OR

$-s$ such that $f(s)=\widetilde{s}$.

Verifier's output: $V$ accepts if the proof in stage 3 succeeds; otherwise, it rejects.

Fig. 2. The simpler variant of our protocol: Simple-c $\mathcal{Z K}$.

We define $\mathbf{L}_{\text {sim }}$ and $\mathbf{L}_{\text {sim }}^{a}$ to be the languages corresponding to $\mathbf{R}_{\text {sim }}$ and $\mathbf{R}_{\text {sim }}^{a}$ respectively. Note that for every polynomial $a$, it holds that $\mathbf{L}_{\text {sim }}^{a} \in \mathbf{N P}$. We say that $\mathcal{Z}=\left\{\mathcal{Z}_{n}\right\}$ is a hard distribution over the statements of $\mathbf{L}_{\text {sim }}^{a}$ if there exists a negligible function negl such that for every non-uniform PPT algorithm $A^{*}$ and every sufficiently large $n$ it holds that $\operatorname{Pr}\left[\lambda \leftarrow \mathcal{Z}_{n} ; \omega \leftarrow A^{*}\left(1^{n}, \lambda\right) ; \mathbf{R}_{\text {sim }}^{a}(\lambda, \omega)=\right.$ $1] \leq \operatorname{negl}(n)$.

The main result of this section is the following theorem.

Theorem 1. Assume the existence of collision-resistant hash functions and injective one-way functions. Further, public-coin differing-inputs obfuscation (pcdiO) for the class of all polynomial-size Turing machines that halt in a polynomial number of steps 8 Then, there exists a constant round, fully concurrent zero-knowledge protocol with negligible soundness, for all languages in NP.

We prove the above theorem by proving that protocol Simple-c $\mathcal{Z} \mathcal{K}$ is a fully concurrent zero-knowledge protocol with negligible soundness error. It is clear that the protocol has constant rounds and perfect completeness. We have already

${ }^{8}$ We note that we actually do not need obfuscation for the class of all PPT Turing machines. Instead, we only need obfuscation for those Turing machines of the form SimLock ${ }^{a}$ where $a$ is a polynomial and SimLock ${ }^{a}$ is the same as SimLock except that it runs for at most $a(|x|)$ steps on input $x$. 
discussed briefly the main ideas for proving the soundness and concurrent ZK of this protocol. We discuss a few more points here and provide the full proofs in PPS15.

To prove the soundness, we start by proving some claims about the obfuscation of Turing machine SimLock ${ }_{\lambda, s}$. In Section 6 we prove that it is hard for any (nonuniform) prover $P^{*}$ to write a "fake witness" $\omega$ to the statements $\lambda$ sampled using the preamble GenStat (see lemma 1). Using this lemma, we show that a sampling algorithm that outputs the pair of machines $\left(\operatorname{SimLock}_{\lambda, s}, \operatorname{SimLock}_{\lambda, 0^{n}}\right)$ is a nice sampler for Turing machines - which, roughly speaking, means that it is hard to produce an input $y$ such that $\operatorname{SimLock}_{\lambda, s}(y) \neq \operatorname{SimLock}_{\lambda, 0^{n}}(y)$. Therefore, by security of pc-diO, the obfuscation of SimLock ${ }_{\lambda, s}$ will be indistinguishable from that of $\operatorname{SimLock}_{\lambda, 0^{n}}$. This however requires some care since we have to ensure that $\lambda$ can indeed be correctly sampled using public-coins. But $\lambda$ consists of $(h, r)$ which are completely random strings, and $c$ is the output of $P^{*}$ which is a publicly known deterministic TM. Therefore we can actually sample $\lambda$ and still ensure hardness of finding a differing-input given $(h, r)$. Now, the soundness follows by considering three hybrids as before and violating the hardness of oneway functions (similar to the soundness of standalone ZK in section 1.1). The full proof appears in PPS15.

To prove concurrent ZK we consider two simulators. The first one is called the internal simulator which requires access to an inversion oracle $\mathcal{I}$ for (injective) one-way functions. The second is the main simulator which essentially runs exactly as the internal simulator (by committing its description in $c$ ) and extracting the secrets using the obfuscated programs. The full descriptions of both the simulators as well as the full proof of indistinguishability of simulation are given in PPS15.

An important issue that we did not discuss relates to the randomness used in the simulation. For the simulation to work, it is essential the internal simulator and the main simulator must use identical randomness in computing the messages that are "fed" to the verifier. This creates a circularity in the security proof: how can the commitments sent by the main simulator be "secure" when the message in the commitment (i.e., the program of the internal simulator) is correlated to the randomness used to create the commitment. We address this issue as follows: we do not include the randomness as part of the internal simulator's description in the "plain;" instead, we include it in the "committed" form using a perfectly binding commitment which can be recovered using the inversion oracle $\mathcal{I}$ - e.g., using commitments based on hard-core bits GL89.

\section{The Four Round Construction}

In the previous section, we presented a reduction from constant round, concurrent zero-knowledge to diO based on standard cryptographic assumptions. In this section, we present a similar reduction for four message concurrent zeroknowledge.

Let us start by optimizing the number of rounds in our constant round protocol of previous section. The standalone ZK protocol used in stage 2 has at least 
four rounds 9 Since the last message of this ZK protocol must come from the verifier, our resulting protocol will have at least five rounds even after optimizations.

We consider two approaches to obtain a four round protocol. First, we can use a two-round ZK protocol with super polynomial time simulation Pas03. This approach gives us a reduction where the soundness of the resulting protocol must assume sub-exponential hardness assumptions. The second approach is to use a $\mathrm{WI}$ protocol to prove the correctness of the obfuscated program. However, in typical applications of WI, to get any useful security we must somehow ensure that the statement being proven has at least two witnesses.

The standard approach in such cases is to consider two independently sampled statements, in this case, two obfuscated programs $\widetilde{M}_{\lambda, s}$ and $\widetilde{M}_{\lambda, s^{\prime}}$; and prove that at least one of them is correctly constructed using a WI proof. However, this approach actually fails for a very interesting reason. Although it does hide one of the secrets $s, s^{\prime}$, it actually breaks the simulation. Indeed, the internal simulator committed to in the preamble, will have no efficient way of knowing which of these two programs is actually correctly prepared. In particular, it will have to ask for the inversion of two challenges per session but the main simulator might be able to return only one of them (since one of the obfuscated programs could have been maliciously prepared). Attempting to overcome this subtle issue actually breaks the hardness of $\mathbf{R}_{\text {sim }}$.

We therefore use a different approach; we set up an "intermediate statement" which is selected by the prover, and require the prover to provide a WIPOK of its correctness. The verifier then proves that either this intermediate statement is true or the obfuscated program is correctly prepared. The intermediate statement is prepared in such a way that it is possible to make it false and succeed (using the real witness for $x$ ) without the verifier noticing. This allows us to ensure that the obfuscated program must be correctly prepared and simulation still continues to go through. For the soundness, roughly speaking, we can extract the witness corresponding to the "intermediate statement" by using the extractor of WIPOK; we then use it to simulate the WI proof that comes from verifier's side. This allows us to again enforce the ideas we developed to prove the soundness of the Simple-c $\mathcal{Z} \mathcal{K}$ protocol.

To setup the "intermediate statement" we use perfectly binding commitments to specially prepared strings. In the final proof, we will need to actually extract the secret $s$ to violate the hardness of one-way functions. We get around this difficulty by using a combination of the WIPOK used by the prover and a ZAP proof. We now present a sketch of our four round protocol below. The formal description of the protocol appears [PPS15.

Four round protocol for concurrent zero-knowledge. The protocol has four components whose messages will be sent in parallel:

${ }^{9}$ To keep our reduction from concurrent ZK to obfuscation free from "knowledge assumptions," we cannot use 3-round ZK protocols based on such assumptions. 
1. The first component is the GenStat protocol, producing statements of the form $\lambda=\langle h, c, r\rangle$.

2. The second component is a three round WIPOK given by the prover to the verfier. The prover prepares two commitments, namely $\widetilde{t}_{1}=\operatorname{Com}\left(0 \| t_{1} ; v_{1}\right)$ and $\widetilde{t}_{2}=\operatorname{Com}\left(0 \| t_{2} ; v_{2}\right)$ and proves that either $\left(\widetilde{t}_{1}, \widetilde{t}_{2}\right)$ are correctly prepared or $x$ is true. The 3 messages of this WIPOK will be denoted by $\langle\alpha, \beta, \gamma\rangle$.

3. The final component is a ZAP for a specially prepared statement, which will let us extract either a witness to $x$ or the secret $s$ in the proof of soundness. The special statement is prepared as follows.

The prover creates two commitments $\tau_{1}, \tau_{2}$ such that $\tau_{1}$ uses string $t_{1}$ (defined above in item 2) as its randomness; likewise $\tau_{2}$ uses $t_{2}$. Further, the value committed to in one of them is the witness $w$ for statement $x$. The prover then proves, using a ZAP, that there exists $i \in\{1,2\}$ such that $\tau_{i}$ is a commitment to $w$ using $t_{i}$. The two messages of this ZAP are denoted by $\left\langle\sigma^{\prime}, \pi^{\prime}\right\rangle$.

To get four rounds, the messages of these components are piggy backed with each other. The main result of this section is the following theorem.

Theorem 2. Assume the existence of collision-resistant hash functions and trapdoor one-way permutations (alternatively, injective one-way functions and ZAP proofs for $\mathbf{N P}$ ). Further, for every polynomial $a: \mathbb{N} \rightarrow \mathbb{N}$, and every hard distribution $\mathcal{Z}$ over the statements of $\mathbf{L}_{\text {sim }}^{a}$, assume the existence of $\mathcal{Z}$-auxiliary differing-input obfuscation (diO) for the class of all polynomial-size Turing machines that halt in a polynomial number of steps. Then, there exists a four message, fully concurrent zero-knowledge protocol with negligible soundness, for all languages in NP.

We have already discussed main ideas behind the proof of soundness and concurrent zero-knowledge of this protocol. The full details are given in PPS15.

\section{References}

$\left[\mathrm{ABG}^{+} 13\right]$ Wee, H.: On obfuscating point functions. In: STOC, pp. 523-532 (2005)

[App13] Applebaum, B.: Bootstrapping obfuscators via fast pseudorandom functions. Cryptology ePrint Archive, Report 2013/699 (2013), http://eprint.iacr.org/2013/699.pdf

[Bar01] Barak, B.: How to go beyond the black-box simulation barrier. In: FOCS, pp. 106-115 (2001)

[Bar02] Barak, B.: Constant-round coin-tossing with a man in the middle or realizing the shared random string model. In: FOCS (2002)

$\left[\mathrm{BBC}^{+} 14\right]$ Barak, B., Bitansky, N., Canetti, R., Kalai, Y.T., Paneth, O., Sahai, A.: Obfuscation for evasive functions. In: Lindell, Y. (ed.) TCC 2014. LNCS, vol. 8349, pp. 26-51. Springer, Heidelberg (2014)

[BC10] Bitansky, N., Canetti, R.: On strong simulation and composable point obfuscation. In: Rabin, T. (ed.) CRYPTO 2010. LNCS, vol. 6223, pp. 520-537. Springer, Heidelberg (2010) 
[BCCT13] Bitansky, N., Canetti, R., Chiesa, A., Tromer, E.: Recursive composition and bootstrapping for snarks and proof-carrying data. In: STOC, pp. 111-120 (2013)

$\left[\mathrm{BCG}^{+} 11\right]$ Bitansky, N., Canetti, R., Goldwasser, S., Halevi, S., Kalai, Y.T., Rothblum, G.N.: Program obfuscation with leaky hardware. In: Lee, D.H., Wang, X. (eds.) ASIACRYPT 2011. LNCS, vol. 7073, pp. 722-739. Springer, Heidelberg (2011)

[BCP14] Boyle, E., Chung, K.-M., Pass, R.: On extractability obfuscation. In: Lindell, Y. (ed.) TCC 2014. LNCS, vol. 8349, pp. 52-73. Springer, Heidelberg (2014), Preliminary version on Eprint 2013: http://eprint.iacr.org/2013/650.pdf

[BCPR13] Bitansky, N., Canetti, R., Paneth, O., Rosen, A.: More on the impossibility of virtual-black-box obfuscation with auxiliary input. Cryptology ePrint Archive, Report 2013/701 (2013), http://eprint.iacr.org/2013/701.pdf

[BFM88] Blum, M., Feldman, P., Micali, S.: Non-interactive zero-knowledge and its applications (extended abstract). In: STOC, pp. 103-112 (1988)

[BG92] Bellare, M., Goldreich, O.: On defining proofs of knowledge. In: Brickell, E.F. (ed.) CRYPTO 1992. LNCS, vol. 740, pp. 390-420. Springer, Heidelberg (1993)

[BG02] Barak, B., Goldreich, O.: Universal arguments and their applications. In: Annual IEEE Conference on Computational Complexity (CCC), vol. 17 (2002), Preliminary full version available as Cryptology ePrint Archive, Report 2001/105.

[BGGL01] Barak, B., Goldreich, O., Goldwasser, S., Lindell, Y.: Resettably-sound zero-knowledge and its applications. In: FOCS 2001, pp. 116-125 (2001)

[BGI $\left.{ }^{+} 01\right]$ Barak, B., Goldreich, O., Impagliazzo, R., Rudich, S., Sahai, A., Vadhan, S.P., Yang, K.: On the (im)possibility of obfuscating programs. In: Kilian, J. (ed.) CRYPTO 2001. LNCS, vol. 2139, pp. 1-18. Springer, Heidelberg (2001)

[BGK $\left.{ }^{+} 13\right]$ Barak, B., Garg, S., Kalai, Y.T., Paneth, O., Sahai, A.: Protecting obfuscation against algebraic attacks. IACR Cryptology ePrint Archive, 2013:631 (2013)

[BGT14] Bitansky, N., Garg, S., Telang, S.: Succinct randomized encodings and their applications. Cryptology ePrint Archive, Report 2014/771 (2014), http://eprint.iacr.org/

[BL04] Barak, B., Lindell, Y.: Strict polynomial-time in simulation and extraction. SIAM Journal on Computing 33(4), 783-818 (2004), Extended abstract appeared in STOC 2002

[Blu87] Blum, M.: How to prove a theorem so no one else can claim it. In: Proceedings of the International Congress of Mathematicians, pp. 1444-1451 (1987)

[BOV03] Barak, B., Ong, S.J., Vadhan, S.P.: Derandomization in cryptography. In: Boneh, D. (ed.) CRYPTO 2003. LNCS, vol. 2729, pp. 299-315. Springer, Heidelberg (2003)

[BP04] Bellare, M., Palacio, A.: The knowledge-of-exponent assumptions and 3-round zero-knowledge protocols. In: Franklin, M. (ed.) CRYPTO 2004. LNCS, vol. 3152, pp. 273-289. Springer, Heidelberg (2004)

[BP12a] Bitansky, N., Paneth, O.: From the impossibility of obfuscation to a new non-black-box simulation technique. In: FOCS, pp. 223-232 (2012) 
[BP12b] Bitansky, N., Paneth, O.: Point obfuscation and 3-round zero-knowledge. In: Cramer, R. (ed.) TCC 2012. LNCS, vol. 7194, pp. 190-208. Springer, Heidelberg (2012)

[BP13a] Bitansky, N., Paneth, O.: On the impossibility of approximate obfuscation and applications to resettable cryptography. In: STOC, pp. 241-250 (2013)

[BP13b] Boyle, E., Pass, R.: Limits of extractability assumptions with distributional auxiliary input. Cryptology ePrint Archive, Report 2013/703 (2013), http://eprint.iacr.org/2013/703.pdf

[BR13a] Brakerski, Z., Rothblum, G.N.: Obfuscating conjunctions. In: Canetti, R., Garay, J.A. (eds.) CRYPTO 2013, Part II. LNCS, vol. 8043, pp. 416-434. Springer, Heidelberg (2013)

[BR13b] Brakerski, Z., Rothblum, G.N.: Virtual black-box obfuscation for all circuits via generic graded encoding. Cryptology ePrint Archive, Report 2013/563 (2013), http://eprint.iacr.org/2013/563.pdf

[BSMP91] Blum, M., De Santis, A., Micali, S., Persiano, G.: Noninteractive zeroknowledge. SIAM J. Comput. 20(6), 1084-1118 (1991)

[BZ13] Boneh, D., Zhandry, M.: Multiparty key exchange, efficient traitor tracing, and more from indistinguishability obfuscation. Cryptology ePrint Archive, Report 2013/642 (2013), http://eprint.iacr.org/

[CD09] Canetti, R., Dakdouk, R.R.: Towards a theory of extractable functions. In: Reingold, O. (ed.) TCC 2009. LNCS, vol. 5444, pp. 595-613. Springer, Heidelberg (2009)

[CGGM00] Canetti, R., Goldreich, O., Goldwasser, S., Micali, S.: Resettable zeroknowledge. In: Proc. 32th STOC, pp. 235-244 (2000)

[CHJV14] Canetti, R., Holmgren, J., Jain, A., Vaikuntanathan, V.: Indistinguishability obfuscation of iterated circuits and ram programs. Cryptology ePrint Archive, Report 2014/769 (2014), http://eprint.iacr.org/

[CKPR03] Canetti, R., Kilian, J., Petrank, E., Rosen, A.: Black-box concurrent zeroknowledge requires (almost) logarithmically many rounds. SIAM Journal on Computing 32(1), 1-47 (2003), Preliminary version in STOC 2001

[CLP10] Canetti, R., Lin, H., Pass, R.: Adaptive hardness and composable security in the plain model from standard assumptions. In: FOCS, pp. 541-550 (2010), Full version: http://www.cs.cornell.edu/ rafael/papers/ccacommit.pdf

[CLP13a] Canetti, R., Lin, H., Paneth, O.: Public-coin concurrent zero-knowledge in the global hash model. In: Sahai, A. (ed.) TCC 2013. LNCS, vol. 7785, pp. 80-99. Springer, Heidelberg (2013)

[CLP13b] Chung, K.-M., Lin, H., Pass, R.: Constant-round concurrent zero knowledge from p-certificates. In: FOCS (2013)

[COPV13] Chung, K.-M., Ostrovsky, R., Pass, R., Visconti, I.: Simultaneous resettability from one-way functions. In: FOCS, pp. 231-240 (2013)

[CPS13] Chung, K.-M., Pass, R., Seth, K.: Non-black-box simulation from one-way functions and applications to resettable security. In: STOC, pp. 231-240 (2013)

[CRV10] Canetti, R., Rothblum, G.N., Varia, M.: Obfuscation of hyperplane membership. In: Micciancio, D. (ed.) TCC 2010. LNCS, vol. 5978, pp. 72-89. Springer, Heidelberg (2010)

[CV13] Canetti, R., Vaikuntanathan, V.: Obfuscating branching programs using black-box pseudo-free groups. IACR Cryptology ePrint Archive, 2013:500 (2013) 
[Dam91] Damgård, I.B.: Towards practical public key systems secure against chosen ciphertext attacks. In: Feigenbaum, J. (ed.) CRYPTO 1991. LNCS, vol. 576, pp. 445-456. Springer, Heidelberg (1992)

[Dam00] Damgård, I.B.: Efficient concurrent zero-knowledge in the auxiliary string model. In: Preneel, B. (ed.) EUROCRYPT 2000. LNCS, vol. 1807, pp. 418-430. Springer, Heidelberg (2000)

[DGS09] Deng, Y., Goyal, V., Sahai, A.: Resolving the simultaneous resettability conjecture and a new non-black-box simulation strategy. In: FOCS (2009)

[DL07] Deng, Y., Lin, D.: Instance-dependent verifiable random functions and their application to simultaneous resettability. In: Naor, M. (ed.) EUROCRYPT 2007. LNCS, vol. 4515, pp. 148-168. Springer, Heidelberg (2007)

[DN00] Dwork, C., Naor, M.: Zaps and their applications. In: Proc. 41st FOCS, pp. 283-293 (2000)

[DNS98] Dwork, C., Naor, M., Sahai, A.: Concurrent zero knowledge. In: Proc. 30th STOC, pp. 409-418 (1998)

[DS98] Dwork, C., Sahai, A.: Concurrent zero-knowledge: Reducing the need for timing constraints. In: Krawczyk, H. (ed.) CRYPTO 1998. LNCS, vol. 1462, pp. 442-457. Springer, Heidelberg (1998)

[FFS88] Feige, U., Fiat, A., Shamir, A.: Zero-knowledge proofs of identity. Journal of Cryptology 1(2), 77-94 (1987), Preliminary version in STOC 1987

[FLS99] Feige, Lapidot, Shamir.: Multiple noninteractive zero knowledge proofs under general assumptions. SIAM Journal on Computing 29 (1999)

[FS89] Feige, U., Shamir, A.: Zero knowledge proofs of knowledge in two rounds. In: Brassard, G. (ed.) CRYPTO 1989. LNCS, vol. 435, pp. 526-544. Springer, Heidelberg (1990)

[FS90] Feige, U., Shamir, A.: Witness indistinguishable and witness hiding protocols. In: Proc. 22nd STOC, pp. 416-426 (1990)

[GGH $\left.{ }^{+} 13\right]$ Garg, S., Gentry, C., Halevi, S., Raykova, M., Sahai, A., Waters, B.: Candidate indistinguishability obfuscation and functional encryption for all circuits. In: FOCS (2013)

[GGHW14] Garg, S., Gentry, C., Halevi, S., Wichs, D.: On the implausibility of differing-inputs obfuscation and extractable witness encryption with auxiliary input. In: Garay, J.A., Gennaro, R. (eds.) CRYPTO 2014, Part I. LNCS, vol. 8616, pp. 518-535. Springer, Heidelberg (2014)

[GIS ${ }^{+}$10] Goyal, V., Ishai, Y., Sahai, A., Venkatesan, R., Wadia, A.: Founding cryptography on tamper-proof hardware tokens. In: Micciancio, D. (ed.) TCC 2010. LNCS, vol. 5978, pp. 308-326. Springer, Heidelberg (2010)

[GJO $\left.{ }^{+} 13\right]$ Goyal, V., Jain, A., Ostrovsky, R., Richelson, S., Visconti, I.: Concurrent zero knowledge in the bounded player model. In: Sahai, A. (ed.) TCC 2013. LNCS, vol. 7785, pp. 60-79. Springer, Heidelberg (2013)

[GJS11] Garg, S., Jain, A., Sahai, A.: Leakage-resilient zero knowledge. In: Rogaway, P. (ed.) CRYPTO 2011. LNCS, vol. 6841, pp. 297-315. Springer, Heidelberg (2011), Full version at: http://www.cs.ucla.edu/ abhishek/papers/lrzk.pdf

[GK96] Goldreich, O., Krawczyk, H.: On the composition of zero-knowledge proof systems. SIAM Journal on Computing 25(1), 169-192 (1996), Preliminary version appeared in Paterson, M. (ed.): ICALP 1990. LNCS, vol. 443, pp. 268-282. Springer, Heidelberg (1990)

[GK05] Goldwasser, S., Kalai, Y.T.: On the impossibility of obfuscation with auxiliary input. In: FOCS, pp. 553-562 (2005) 
[GK13] Goldwasser, S., Kalai, Y.T.: A note on the impossibility of obfuscation with auxiliary input. Cryptology ePrint Archive, Report 2013/665 (2013), http://eprint.iacr.org/2013/665.pdf

[GL89] Goldreich, O., Levin, L.A.: A hard-core predicate for all one-way functions. In: Proc. 21st STOC, pp. 25-32 (1989)

$\left[\mathrm{GLP}^{+} 15\right]$ Goyal, V., Lin, H., Pandey, O., Pass, R., Sahai, A.: Round-efficient concurrently composable secure computation via a robust extraction lemma. In: TCC (2015), Full version of this work available as IACR Eprint Report $2012 / 65$

[GM11] Goyal, V., Maji, H.K.: Stateless cryptographic protocols. In: FOCS, pp. 678-687 (2011)

[GMR85] Goldwasser, S., Micali, S., Rackoff, C.: The knowledge complexity of interactive proof-systems. In: Proc. 17th STOC, pp. 291-304. ACM (1985)

[Gol02] Goldreich, O.: Concurrent zero-knowledge with timing, revisited. In: Proc. 34th STOC, pp. 332-340 (2002)

[GOS06] Groth, J., Ostrovsky, R., Sahai, A.: Non-interactive zaps and new techniques for NIZK. In: Dwork, C. (ed.) CRYPTO 2006. LNCS, vol. 4117, pp. 97-111. Springer, Heidelberg (2006)

[Goy13] Goyal, V.: Non-black-box simulation in the fully concurrent setting. In: STOC, pp. 221-230 (2013)

[GR07] Goldwasser, S., Rothblum, G.N.: On best-possible obfuscation. In: Vadhan, S.P. (ed.) TCC 2007. LNCS, vol. 4392, pp. 194-213. Springer, Heidelberg (2007)

[GS12a] Garg, S., Sahai, A.: Adaptively secure multi-party computation with dishonest majority. In: Safavi-Naini, R., Canetti, R. (eds.) CRYPTO 2012. LNCS, vol. 7417, pp. 105-123. Springer, Heidelberg (2012)

[GS12b] Gupta, D., Sahai, A.: On constant-round concurrent zero-knowledge from a knowledge assumption. CoRR, abs/1210.3719 (2012)

[Had00] Hada, S.: Zero-knowledge and code obfuscation. In: Okamoto, T. (ed.) ASIACRYPT 2000. LNCS, vol. 1976, pp. 443-457. Springer, Heidelberg (2000)

[Had10] Hada, S.: Secure obfuscation for encrypted signatures. In: Gilbert, H. (ed.) EUROCRYPT 2010. LNCS, vol. 6110, pp. 92-112. Springer, Heidelberg (2010)

[HRSV07] Hohenberger, S., Rothblum, G.N., Shelat, A., Vaikuntanathan, V.: Securely obfuscating re-encryption. In: Vadhan, S.P. (ed.) TCC 2007. LNCS, vol. 4392, pp. 233-252. Springer, Heidelberg (2007)

[HSW13] Hohenberger, S., Sahai, A., Waters, B.: Replacing a random oracle: Full domain hash from indistinguishability obfuscation. In: Cryptology ePrint Archive, Report 2013/509 (2013),

http://eprint.iacr.org/2013/509.pdf

[HT99] Hada, S., Tanaka, T.: On the existence of 3-round zero-knowledge protocols. Cryptology ePrint Archive, Report 1999/009 (1999), http://eprint.iacr.org/

[IPS15] Ishai, Y., Pandey, O., Sahai, A.: Public Coin Differing-Inputs Obfuscation. In: TCC (2015), Cryptology Eprint Archive Report 2014/942

[Kil92] Kilian, J.: A note on efficient zero-knowledge proofs and arguments (extended abstract). In: Proc. 24th STOC, pp. 723-732 (1992)

[Kil95] Kilian, J.: Improved efficient arguments. In: Coppersmith, D. (ed.) CRYPTO 1995. LNCS, vol. 963, pp. 311-324. Springer, Heidelberg (1995) 
[KP01] Kilian, J., Petrank, E.: Concurrent and resettable zero-knowledge in polylogarithm rounds. In: STOC, pp. 560-569 (2001)

[KPR98] Kilian, J., Petrank, E., Rackoff, C.: Lower bounds for zero knowledge on the Internet. In: Proc. 39th FOCS, pp. 484-492 (1998)

[KRW13] Koppula, V., Ramchen, K., Waters, B.: Separations in circular security for arbitrary length key cycles. Cryptology ePrint Archive, Report 2013/683 (2013), http://eprint.iacr.org/2013/683.pdf

[LP14] Lin, H., Pass, R.: Succinct garbling schemes and applications. Cryptology ePrint Archive, Report 2014/766 (2014), http://eprint.iacr.org/

[LPS04] Lynn, B., Prabhakaran, M., Sahai, A.: Positive results and techniques for obfuscation. In: Cachin, C., Camenisch, J.L. (eds.) EUROCRYPT 2004. LNCS, vol. 3027, pp. 20-39. Springer, Heidelberg (2004)

[Mic94] Micali, S.: CS proofs. In: Proc. 35th FOCS, pp. 436-453 (1994)

[MO13] Marcedone, A., Orlandi, C.: Obfuscation $==$ (ind-cpa security $=/=$ circular security). Cryptology ePrint Archive, Report 2013/690 (2013), http://eprint.iacr.org/2013/690.pdf

[MR13] Moran, T., Rosen, A.: There is no indistinguishability obfuscation in pessiland. Cryptology ePrint Archive, Report 2013/643 (2013), http://eprint.iacr.org/2013/643.pdf

[MRV99] Micali, S., Rabin, M.O., Vadhan, S.P.: Verifiable random functions. In: FOCS, pp. 120-130 (1999)

[Nao89] Naor, M.: Bit commitment using pseudo-randomness (extended abstract). In: Brassard, G. (ed.) CRYPTO 1989. LNCS, vol. 435, pp. 128-136. Springer, Heidelberg (1990)

[Pan14] Pandey, O.: Achieving constant round leakage-resilient zero-knowledge. In: Lindell, Y. (ed.) TCC 2014. LNCS, vol. 8349, pp. 146-166. Springer, Heidelberg (2014), Preliminary version on Eprint 2012: http://eprint.iacr.org/2012/362.pdf

[Pas03] Pass, R.: Simulation in quasi-polynomial time, and its application to protocol composition. In: Biham, E. (ed.) EUROCRYPT 2003. LNCS, vol. 2656, pp. 160-176. Springer, Heidelberg (2003)

[Pas04] Pass, R.: Bounded-concurrent secure multi-party computation with a dishonest majority. In: Proc. 36th STOC, pp. 232-241 (2004)

[PPS15] Pandey, O., Prabhakaran, M., Sahai, A.: Obfuscation-based non-blackbox simulation and four message concurrent zero knowledge for np. In: TCC 2015 (2015), Full version of this work available as Cryptology ePrint Archive Report 2013/754

[PR03] Pass, R., Rosen, A.: Bounded-concurrent secure two-party computation in a constant number of rounds. In: Proc. 44th FOCS (2003)

[PR05a] Pass, R., Rosen, A.: Concurrent non-malleable commitments. In: FOCS (2005)

[PR05b] Pass, R., Rosen, A.: New and improved constructions of non-malleable cryptographic protocols. In: STOC (2005)

[PRS02] Prabhakaran, M., Rosen, A., Sahai, A.: Concurrent zero knowledge with logarithmic round-complexity. In: FOCS (2002)

[PTV10] Pass, R., Tseng, W.-L.D., Venkitasubramaniam, M.: Eye for an eye: Efficient concurrent zero-knowledge in the timing model. In: Micciancio, D. (ed.) TCC 2010. LNCS, vol. 5978, pp. 518-534. Springer, Heidelberg (2010)

[PV08] Pass, R., Venkitasubramaniam, M.: On constant-round concurrent zeroknowledge. In: Canetti, R. (ed.) TCC 2008. LNCS, vol. 4948, pp. 553-570. Springer, Heidelberg (2008) 
[RK99] Richardson, R., Kilian, J.: On the concurrent composition of zeroknowledge proofs. In: Stern, J. (ed.) EUROCRYPT 1999. LNCS, vol. 1592, pp. 415-432. Springer, Heidelberg (1999)

[Ros00] Rosen, A.: A note on the round-complexity of concurrent zero-knowledge. In: Bellare, M. (ed.) CRYPTO 2000. LNCS, vol. 1880, pp. 451-468. Springer, Heidelberg (2000)

[Ros04] Rosen, A.: The Round-Complexity of Black-Box Concurrent ZeroKnowledge. PhD thesis, Department of Computer Science and Applied Mathematics, Weizmann Institute of Science, Rehovot, Israel (2004)

[SW13] Sahai, A., Waters, B.: How to use indistinguishability obfuscation: Deniable encryption, and more. IACR Cryptology ePrint Archive, 2013:454 (2013)

[TW87] Tompa, M., Woll, H.: Random self-reducibility and zero-knowledge interactive proofs of possession of information. In: Proc. 28th FOCS, pp. 472-482 (1987)

[Wee05] Wee, H.: On obfuscating point functions. In: STOC, pp. 523-532 (2005)

\section{Hardness of GenStat and a Nice Sampler}

In this section, we prove that a randomly sampled transcript of GenStat is a hard distribution over the statements of $\mathbf{L}_{\text {sim }}^{a}$ for every polynomial $a$. Recall that $\mathcal{Z}=$ $\left\{\mathcal{Z}_{n}\right\}$ is a hard distribution over the statements of $\mathbf{L}_{\text {sim }}^{a}$ if there exists a negligible function negl such that for every non-uniform PPT algorithm $A^{*}$ and every sufficiently large $n$ it holds that $\operatorname{Pr}\left[\lambda \leftarrow \mathcal{Z}_{n} ; \omega \leftarrow A^{*}\left(1^{n}, \lambda\right) ; \mathbf{R}_{\text {sim }}^{a}(\lambda, \omega)=1\right] \leq$ $\operatorname{negl}(n)$. The preamble GenStat, is recalled below. For convenience, we use a noninteractive perfectly binding commitment scheme; the two-round statisticallybinding commitment scheme of [Nao89] also works.

\subsection{Preamble GenStat}

Statement generation protocol. Let $\left\{\mathcal{H}_{n}\right\}$ be a family of collision-resistant hash functions (CRHF) $h \in \mathcal{H}_{n}$ such that $h:\{0,1\}^{*} \rightarrow\{0,1\}^{n}$ and Com be a noninteractive perfectly-binding commitment scheme for $\{0,1\}^{n}$. The statement generation protocol GenStat $:=\left\langle P_{1}, V_{1}\right\rangle$ is a three round protocol between $P_{1}$ and $V_{1}$ which proceeds as follows:

Protocol GenStat : $=\left\langle P_{1}, V_{1}\right\rangle$ :

1. $V_{1}$ sends a random $h \leftarrow \mathcal{H}_{n}$

2. $P_{1}$ sends a commitment $c=\operatorname{Com}\left(0^{n} ; u\right)$ where $u$ is a randomly chosen

3. $V_{1}$ sends a random string $r \leftarrow\{0,1\}^{n}$

The transcript of the protocol is $\lambda:=\langle h, c, r\rangle$.

\subsection{Hardness of GenStat with respect to relation $\mathbf{R}_{\text {sim }}$}

We defined $\mathbf{R}_{\text {sim }}$ in figure 11 Recall that $\mathbf{R}_{\text {sim }}$ is undecidable in polynomial time in general. But our analysis will ensure that $\mathbf{R}_{\text {sim }}$ is tested only on inputs on which $\Pi^{\mathcal{T}}$ does halt (and in fact halts in a polynomial number of steps). To capture this, we defined a bounded variant of this relation, namely $\mathbf{R}_{\text {sim }}^{a}$ for every polynomial $a: \mathbb{N} \rightarrow \mathbb{N}$. 
Relation $\mathbf{R}_{\text {sim }}^{a}$ : Let $a: \mathbb{N} \rightarrow \mathbb{N}$ be a polynomial; relation $\mathbf{R}_{\text {sim }}^{a}$ is identical to $\mathbf{R}_{\text {sim }}$ except that the witness $\left(u, \Pi^{\langle\cdot\rangle}, \mathcal{T}\right)$ satisfies following additional constraints:

1. $|\mathcal{T}| \leq a(n)$,

2. $\Pi^{\mathcal{T}}$ halts in at most $a(n)$ steps.

Note that $\mathbf{R}_{\text {sim }}^{a}$ can be tested in time poly $(a(n))=$ poly $(n) . \mathbf{L}_{\text {sim }}\left(\operatorname{resp} . \mathbf{L}_{\text {sim }}^{a}\right)$ is the language corresponding to relation $\mathbf{R}_{\text {sim }}$ (resp., $\mathbf{R}_{\text {sim }}^{a}$ ) and $\mathbf{L}_{\text {sim }}^{a} \in \mathbf{N P}$.

The following lemma states that it is hard for any PPT machine $P_{1}^{*}$ to compute a witness $\omega$ to statements $\lambda$ when $\lambda$ is the transcript of GenStat between $P_{1}^{*}$ and an honest $V_{1}$. The proof follows [Bar01].

Lemma 1 (Hardness of GenStat). Assume that $\left\{\mathcal{H}_{n}\right\}$ is a family of collisionresistant hash functions against (non-uniform) PPT algorithms. There exists a negligible function negl such that for every (non-uniform) PPT Turing machine $P_{1}^{*}$, the probability that $P_{1}^{*}$, after interacting with an honest $V_{1}$ in protocol GenStat, writes a string $\omega$ on its (private) output tape such that $\mathbf{R}_{\operatorname{sim}}(\lambda, \omega)=1$ is at most $\operatorname{negl}(n)$, where $\lambda$ is the transcript of interaction between $P_{1}^{*}$ and $V_{1}$, and the probability is taken over the randomness of both $P_{1}^{*}$ and $V_{1}$.

Remark. We note that since $P_{1}^{*}$ is polynomial time, it can only write a $\omega$ of polynomial length. However, since we have to consider all polynomial time $P_{1}^{*}$, it is not known in advance how large $\omega$ will be even though it will be of polynomial size.

Proof of lemma 1. Assume, on the contrary, that there exist polynomials $p, q$ and a prover $P_{1}^{*}$ such that $P_{1}^{*}$ takes at most $p(n)$ steps and writes a string $\omega$ on its private output tape such that for infinitely many values of $n, \delta(n) \geq 1 / q(n)$ where $\delta(n)$ is the probability that $\mathbf{R}_{\operatorname{sim}}(\lambda, \omega)=1$ (where $\lambda$ is sampled as defined in the lemma). Now consider the machine $P_{1}^{*}$ in an execution of GenStat and let $(h, c)$ be the first two messages in this interaction. Let the machine $P_{1, h, c}^{*}$ denote the machine $P_{1}^{*}$ whose state has been frozen up to the point where $c$ is sent in this execution. By a standard averaging argument, it follows that with probability at least $\delta / 2$ over the sampling of $(h, c)$ in this interaction, the probability that $P_{1, h, c}^{*}$ writes a valid witness $\omega$ at the end of the interaction is at least $\delta / 2$. We call such $(h, c)$ "good."

The following procedure finds collisions in $h$ provided $(h, c)$ are good: the procedure chooses two random strings $r_{1}, r_{2}$ each of length $n$, feeds $P_{1}^{*}$ with $r_{1}$ and then with $r_{2}$ separately; let $\omega_{i}=\left(u_{i}, \Pi_{i}^{\langle\cdot,\rangle}, \mathcal{T}_{i}\right)$ be the contents of the private output tape of $P_{1, h, c}^{*}$ when fed with string $r_{i}$ for $i \in\{1,2\}$. The procedure outputs $\left(\Pi_{1}, \Pi_{2}\right)$ as the potential collision on $h$.

We claim that the procedure finds collisions in $h$ with noticeable probability as follows. Note that since $(h, c)$ is good, with probability $\delta^{2} / 4$, it holds that $\mathbf{R}_{\text {sim }}\left(\lambda_{i}, \omega_{i}\right)=1$ where $\lambda_{i}=\left(h, c, r_{i}\right)$. Hence, $\Pi_{i}^{\mathcal{T}_{i}}=r_{i}$ and $h\left(\Pi_{1}\right)=h\left(\Pi_{2}\right)$ w.h.p. since $c$ is perfectly binding.

Now, define $\mathcal{I}$ to be an inversion oracle which on input a query of the form $(f, \widetilde{s})$ for $f \in \mathcal{F}_{n}$ and $\widetilde{s} \in \operatorname{Range}(f)$ outputs $s=f^{-1}(\widetilde{s})$. Then, by definition of 
$\mathbf{R}_{\text {sim }}$ (in particular, due to condition 4 in figure 1), we have that the output of $\Pi_{i}^{\mathcal{T}_{i}}$ is the same as that of $\Pi_{i}^{\mathcal{I}}$. I.e., $\Pi_{i}^{\mathcal{I}}$ outputs $r_{i}$. Since $\Pi_{i}^{\mathcal{I}}$ is a deterministic computation, it holds that $\Pi_{1}$ and $\Pi_{2}$ are different programs whenever $r_{1} \neq r_{2}$ (which happens with prob. $1-2^{-n}$ ). Further, since $P_{1}^{*}$ runs in time at most $p(n)$, programs $\Pi_{1}, \Pi_{2}$ are of size at most $p(n)$. Therefore, $\Pi_{1}$ and $\Pi_{2}$ are collisions in $h$, found with probability at least $\frac{\delta^{2}}{4} \cdot\left(1-2^{-n}\right) \geq \delta^{2} / 8$.

It follows that collisions can be found for a noticeable (specifically, at least $\delta / 2$ ) fraction of functions in $\left\{\mathcal{H}_{n}\right\}$ with noticeable probability (specifically, $\delta^{2} / 8$ ). This concludes the proof.

\subsection{A Nice Sampler for TM}

Protocol GenStat allows us to build a (non uniform) sampling algorithm Samp which will be nice according to definition 6 Samp uses the following simple TM, which was defined earlier:

\section{$\operatorname{SimLock}(\lambda, \omega, s)$ :}

Test if $\mathbf{R}_{\text {sim }}(\lambda, \omega)=1$, and if so output $s$;

Else, output the empty string $0^{n}$.

Also, for a fixed $(\lambda, s)$, define $\operatorname{SimLock}_{\lambda, s}(\cdot):=\operatorname{SimLock}(\lambda, \cdot, s)$. Machine SimLock ${ }_{\lambda, s}$ essentially tests whether the input is a valid witness to $\lambda$, and if so outputs the fixed value $s$, and nothing otherwise. Note that it is possible that SimLock takes $2^{n}$ steps on some inputs. However, no such inputs will be returned by any PPT adversary who uses (an obfuscation of) SimLock. Also, w.l.o.g., we assume that $\operatorname{Steps}\left(\operatorname{SimLock}_{\lambda, s_{1}}, \omega\right)=\operatorname{Steps}\left(\operatorname{SimLock}_{\lambda, s_{2}}, \omega\right)$ for every $\lambda, \omega$ and $\left(s_{1}, s_{2}\right) \in\{0,1\}^{n} \times\{0,1\}^{n}$.

The sampler. The sampling algorithm, Samp $P_{1}^{*}$ is defined with respect to an arbitrary PPT interactive TM $P_{1}^{*}$. ITM $P_{1}^{*}$ follows the instructions of GenStat protocol and interacts with algorithm $V_{1}$.

Samp $_{P_{1}^{*}}\left(1^{n} ; z\right)$.

- $z$ is of the form $(h, r, s) \in \mathcal{H}_{n} \times\{0,1\}^{n} \times\{0,1\}^{n}$.

- Sample a random transcript $\lambda$ of GenStat by interacting with $P_{1}^{*}$ honestly by sending $h$ as the first message and $r$ as the third message. Let $c$ be the output of $P_{1}^{*}$ so that $\lambda=(h, c, r)$.

- Output $\left(z, \operatorname{SimLock}_{\lambda, s}\right.$, SimLock $\left._{\lambda, 0^{n}}\right)$

When the third component of $z$ is fixed to a specific $s$, we will denote the sampler by Samp $s, P_{*}^{*}$ to emphasize a fixed $s$. The following lemma is essentially a corollary of lemma 1. It proves a stronger claim by directly about Samp ${ }_{s, P_{*}^{*}}$; it is easy to see that the claim will trivially follow for a random $s$ since it follows for each one of them.

Lemma 2. For every non-uniform PPT TM $P_{1}^{*}$, and every $s \in\{0,1\}^{n}$, Samp $_{s, P_{1}^{*}}$ is a nice sampler for Turing machines (according to definition [6). 
Proof. Observe that the pair $\left(\operatorname{SimLock}_{\lambda, s}, \operatorname{SimLock}_{\lambda, 0^{n}}\right)$ is always a pair of compatible TMs, by definition of SimLock. Now suppose that the second property of definition 6 is not satisfied. Then there exists an $A$, running in time $a(n)$ for some polynomial $a$, which outputs an $x$ with noticeable probability such that $\operatorname{SimLock}_{\lambda, s}(x) \neq \operatorname{SimLock}_{\lambda, 0^{n}}(x)$, and $|x| \leq a(n)$; here the probability is taken over the sampling of $\lambda$.It follows, from the definition of $\operatorname{SimLock}_{\lambda, s}$, that $x$ must be a witness to $\lambda$ and therefore $A$ is a PPT machine which finds witnesses to statements $\lambda \in \mathbf{L}_{\text {sim }}^{a}$ with noticeable probability. We can use $A$ to violate lemma 1 as follows.

Consider the machine $B_{1, s}^{*}$ which incorporates $P_{1}^{*}$ and $A$. It then samples $\lambda$ by routing messages between $P_{1}^{*}$ and an external (honest) $V_{1}$, and returns the output of $A\left(z, \operatorname{SimLock}_{\lambda, s}, \operatorname{SimLock}_{\lambda, 0^{n}}\right)$. It is straightforward to see that $B_{1, s}^{*}$ violates lemma 1 (for every fixed $s$ ). 